\title{
The Unexpected Rapid Intensification of Tropical Cyclones in Moderate Vertical Wind Shear. Part II: Vortex Tilt
}

\author{
David R. Ryglicki, James D. Doyle, Yi Jin, Daniel Hodyss, And Joshua H. Cossuth \\ Naval Research Laboratory, Monterey, California
}

(Manuscript received 19 January 2018, in final form 7 August 2018)

\begin{abstract}
We investigate a class of tropical cyclones (TCs) that undergo rapid intensification (RI) in moderate vertical wind shear through analysis of a series of idealized model simulations. Two key findings derived from observational analysis are that the average $200-850-\mathrm{hPa}$ shear value is $7.5 \mathrm{~m} \mathrm{~s}^{-1}$ and that the TCs displayed coherent cloud structures, deemed tilt-modulated convective asymmetries (TCA), which feature pulses of deep convection with periods of between 4 and $8 \mathrm{~h}$. Additionally, all of the TCs are embedded in an environment that is characterized by shear associated with anticyclones, a factor that limits depth of the strongest environmental winds in the vertical. The idealized TC develops in the presence of relatively shallow environmental wind shear of an anticyclone. An analysis of the TC tilt in the vertical demonstrates that the source of the observed 4-8-h periodicity of the TCAs can be explained by smaller-scale nutations of the tilt on the longer, slower upshear precession. When the environmental wind shear occurs over a deeper layer similar to that of a trough, the TC does not develop. The TCAs are characterized as collections of updrafts that are buoyant throughout the depth of the TC since they rise into a cold anomaly caused by the tilting vortex. At $90 \mathrm{~h}$ into the simulation, RI occurs, and the tilt nutations (and hence the TCAs) cease to occur.
\end{abstract}

\section{Introduction}

Through a series of satellite and model analyses, Ryglicki et al. (2018; hereafter Part I) presented a class of tropical cyclones (TCs) that underwent rapid intensification (RI; $30 \mathrm{kt}$ or $\sim 15 \mathrm{~m} \mathrm{~s}^{-1}$ increase in $24 \mathrm{~h}$ ) in an environment featuring moderate vertical wind shear (5-10 $\mathrm{m} \mathrm{s}^{-1}$; Rios-Berrios and Torn 2017), contrary to climatological expectations of vertical wind shear and RI (i.e., Gray 1968; Zeng et al. 2008; Kaplan et al. 2010; Wang et al. 2015). Members of this TC class include 1997 eastern Pacific (EPAC) Guillermo, 2008 EPAC Hernan, 2008 EPAC Norbert, 2012 EPAC Fabio, 2015 EPAC Hilda, and 2015 northern Atlantic (NATL) Joaquin. On average, these storms developed in $7.5 \mathrm{~m} \mathrm{~s}^{-1}$ of Statistical Hurricane Intensity Prediction System (SHIPS)-analyzed shear (DeMaria et al. 2005), a value that is roughly one to two standard deviations, depending on the specific basin, above the climatological mean for storms that undergo RI (Kaplan et al. 2010). This study explores the tilt in TCs of this class

Corresponding author: David R. Ryglicki, david.ryglicki@nrlmry. navy.mil and its relationship with the convective features seen in Part I.

Strong vertical wind shear is generally thought to be overwhelmingly negative for the development and intensification of TCs (McBride and Zehr 1981; Bender 1997). This view is well reasoned and well supported, given the plethora of previous works-both observations and models-that establish how vertical wind shear both dynamically and thermodynamically affects TC evolution in negative ways. Vertical wind shear can weaken a TC through ventilation of the warm core aloft (Frank and Ritchie 2001; Knaff et al. 2004); through the midlevel entrainment of dry air (Simpson and Riehl 1958; Cram et al. 2007; Tang and Emanuel 2010; Ge et al. 2013); through the interruption of moist inflow at low levels through flushing of the boundary layer with cold pools (Riemer et al. 2010); by upper-level convergence on the upshear side of the storm (Xu and Wang 2013); or via a combination of all of the above as the secondary circulation, in an azimuthal mean sense, is altered negatively at nearly all levels in the storm (Riemer and Laliberté 2015).

A major reason why the secondary circulation is altered negatively is due to the localization of convection in 
a sheared TC, usually downshear left (Reasor et al. 2013). There have been several mechanisms proposed in the literature discussing this convective anomaly (Willoughby et al. 1984; DeMaria 1996; Frank and Ritchie 1999, 2001; Corbosiero and Molinari 2003; DeHart et al. 2014). These proposed mechanisms include a thermally balanced response to tilting (Jones 1995; DeMaria 1996), a compensating low-level vorticity mechanism (Willoughby et al. 1984), upper-level differential vorticity advection (Frank and Ritchie 1999, 2001), or a localized boundary layer spinup due to the tilt (Riemer et al. 2010, 2013). This localization of convection limits the preferred areas of vertical motion, a factor that could potentially limit any of the existing proposed intensification mechanisms, such as the Wind-Induced Surface Heat Exchange theory (WISHE; Rotunno and Emanuel 1987) or the rotating convective paradigm (Smith and Montgomery 2015).

Despite the overwhelming negative influences of vertical wind shear, there are times where variations in the details of the environmental wind profile can change the intensification pathway of TCs. Onderlinde and Nolan (2016), for example, demonstrated how the helical structure of the background wind can alter intensification characteristics. Rappin and Nolan (2012) discussed how shear orientation can affect genesis and subsequent intensification. Finally, Elsberry and Jeffries (1996) and Finocchio et al. (2016) argued that the depth of the environmental flow can have significant impacts on intensification. Of the six "atypical RI" TCs analyzed in Part I, all of them underwent RI in proximity to an upper-level anticyclone. Part I argued that given the structural differences between tropopause cyclonic and anticyclonic potential vorticity (PV) anomalies (Hoskins et al. 1985; Wirth 2001), the strongest environmental winds associated with anticyclones are likely to be confined to a similar layer as the outflow from the TCusually around $200 \mathrm{hPa}$ (Merrill and Velden 1996). Part I also demonstrated that before the eyes appeared in infrared (IR) and water vapor (WV), each storm exhibited what we referred to as a tilt-modulated convective asymmetry (TCA). TCAs, as diagnosed by cloud-top temperatures colder than $-70^{\circ} \mathrm{C}$, are cloud structures that are responsible for approximately $10000-15000-\mathrm{km}^{2}$ changes in cloud cover, that expand upshear, and, most importantly for the purposes of this study, that appear with periods between 4 and $8 \mathrm{~h}$. A series of numerical simulations in this second part are analyzed to quantify further the dynamics and characteristics, including the vertical structure, of this class of TCs that undergo RI in the presence of moderate vertical wind shear. In Part II of this study, the primary objective is to determine the source of the periodicity of the TCAs documented in Part I. Additionally, the structure of TCAs is also explored.
Previous studies (e.g., Barnes et al. 1983; Barnes and Stossmeister 1986; Barnes et al. 1991; Reasor et al. 2000; Houze 2010; Rogers 2010; Moon and Nolan 2015) have established and diagnosed, both through observations and numerical modeling perspectives, a host of convective elements in TCs including outer rainbands, inner rainbands, the eyewall, convective bursts, and vortex Rossby waves. Hazelton et al. (2017), for example, linked convective bursts to low-level vorticity asymmetries. In terms of the schematic from Part I (Fig. 1 from Part I), this manuscript will highlight how simple differences in the wind profile ("A") affect the development and evolution of the TC, including the tilt ("B"), the localization and movement of convection ("C"), and structural changes associated with the tilt ("D").

A description of the numerical model used in this study, its setup, and the TC center-finding algorithms used to diagnose the vortex tilt are presented in section 2. Section 3 describes the general diagnostics of the TC evolutions, such as the intensity and tilt of the sheared simulations. Section 4 explores the evolution of the TCA, including the "vortical" and buoyant structures of convective towers within the TCAs. The rationale for the term TCA is addressed in section 4, namely, that the TCA is a collection of faster-moving individual convective cells localized within a slower-moving region. Section 5 presents a summary and synthesis of the findings herein in addition to addressing future research needed.

\section{Experiment description}

\section{a. Model description}

The model used for this work is the compressible, nonhydrostatic Bryan cloud model (CM1; Bryan and Fritsch 2002) release 18. Minor additions to this version of CM1 are the cosine Coriolis terms in the zonal and vertical momentum equations, stylized as $e$ (Liang and Chan 2005), and the beta plane approximation. A significant addition is the capability to achieve a thermally balanced background when prescribing an environmental flow and its inclusion in the model's advection terms. Further details of the model setup can be found in appendix A.

Four simulations are carried out: one nonsheared control and three sheared cases. For the sheared cases, two different background wind profiles are used. The first is a Gaussian wind profile:

$$
u(z)=u_{0} \exp \left[\frac{-\left(z-z_{p}\right)^{2}}{2 z_{w}^{2}}\right]+u_{b}
$$



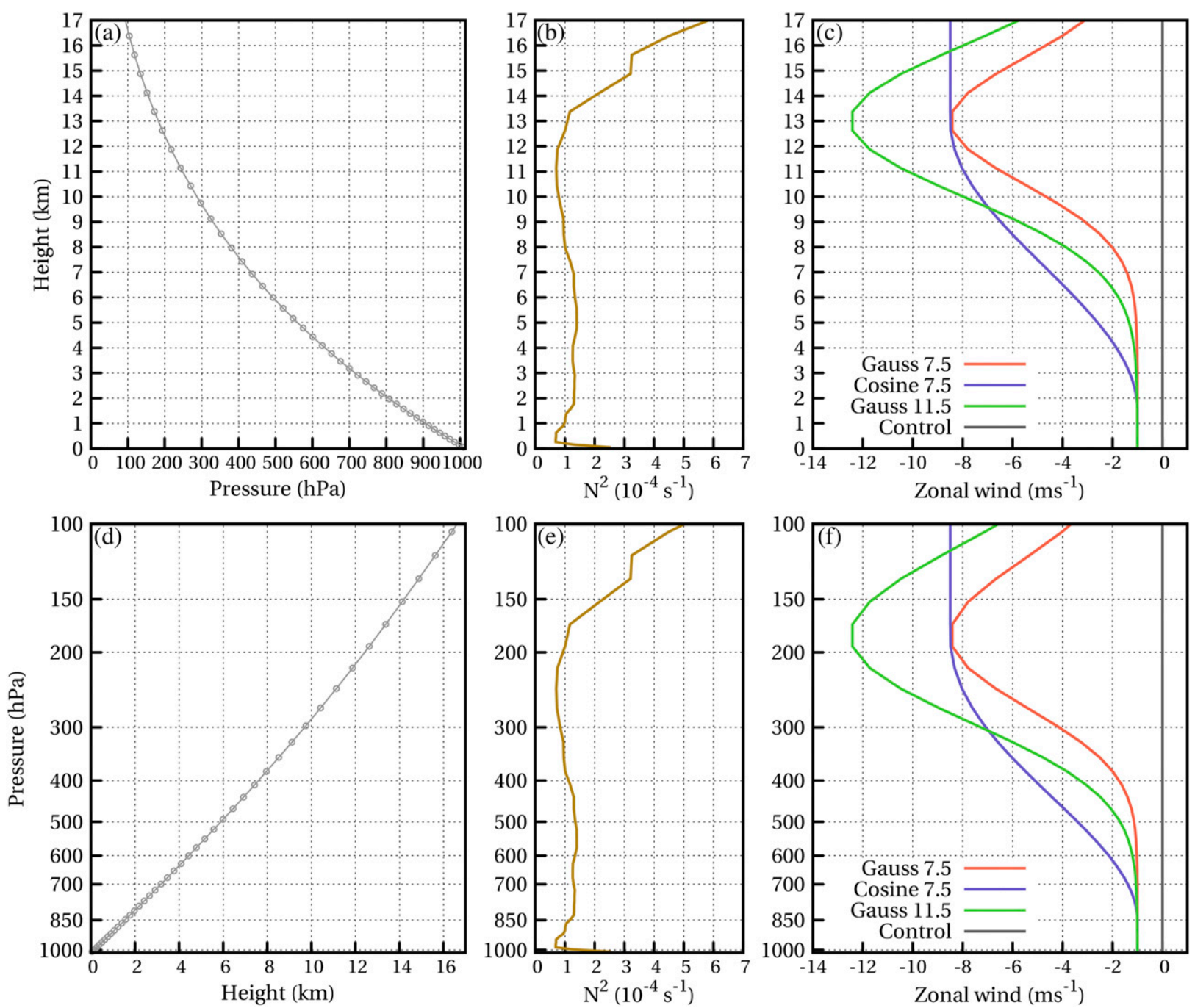

FIG. 1. Background initial conditions vs (top) height and (bottom) pressure of (a),(d) pressure-height; (b),(e) static stability; and (c),(f) winds.

The second is a cosine wind profile:

$$
\begin{aligned}
& u(z)=u_{0}+u_{b}, \quad z \leq z_{\mathrm{st}} \\
& u(z)=u_{0} \cos \left[\pi \frac{\left(z-z_{\mathrm{st}}\right)}{\left(z_{\mathrm{top}}-z_{\mathrm{st}}\right)}\right]+u_{b}, \quad z_{\mathrm{st}}<z<z_{\mathrm{top}} \\
& u(z)=-u_{0}+u_{b}, \quad z_{\mathrm{top}} \leq z
\end{aligned}
$$

More details are found in appendix A. The structure of the background, including the pressure-height relationship, the static stability, and the winds, in both height and pressure coordinates is shown in Fig. 1. The tropopause $\left(N^{2}=2 \times 10^{-4} \mathrm{~s}^{-1}\right)$ is at approximately $14 \mathrm{~km}$ or $150 \mathrm{hPa}$. Since the CM1 uses physical height as its vertical coordinate, the proxy for the $200-850-\mathrm{hPa}$ SHIPS shear used here is $13-1.5 \mathrm{~km}$. Using this methodology, the cosine profile and the weaker Gaussian profile have a shear magnitude of $7.5 \mathrm{~m} \mathrm{~s}^{-1}$, which is approximately the average shear value over the six storms from Part I. Because of the discretization in the CM1, the 13-1.5-km shear values for the two Gaussian profiles are 7.41 and $11.41 \mathrm{~m} \mathrm{~s}^{-1}$, respectively.

The two shear profiles are intended to represent, in a simplified, idealized sense, the depth of an upper-level anticyclone (Gaussian function) and an upper-level trough (cosine function). An example of the differences from the ERA-Interim dataset (Dee et al. 2011) is shown in Fig. 2 (which is also Fig. 9 from Part I): those of 2015 NATL Hurricane Joaquin, one of the six storms identified by Part I and also the only one of the storms identified that encountered moderate shear from two different sources in quick succession. During the period 

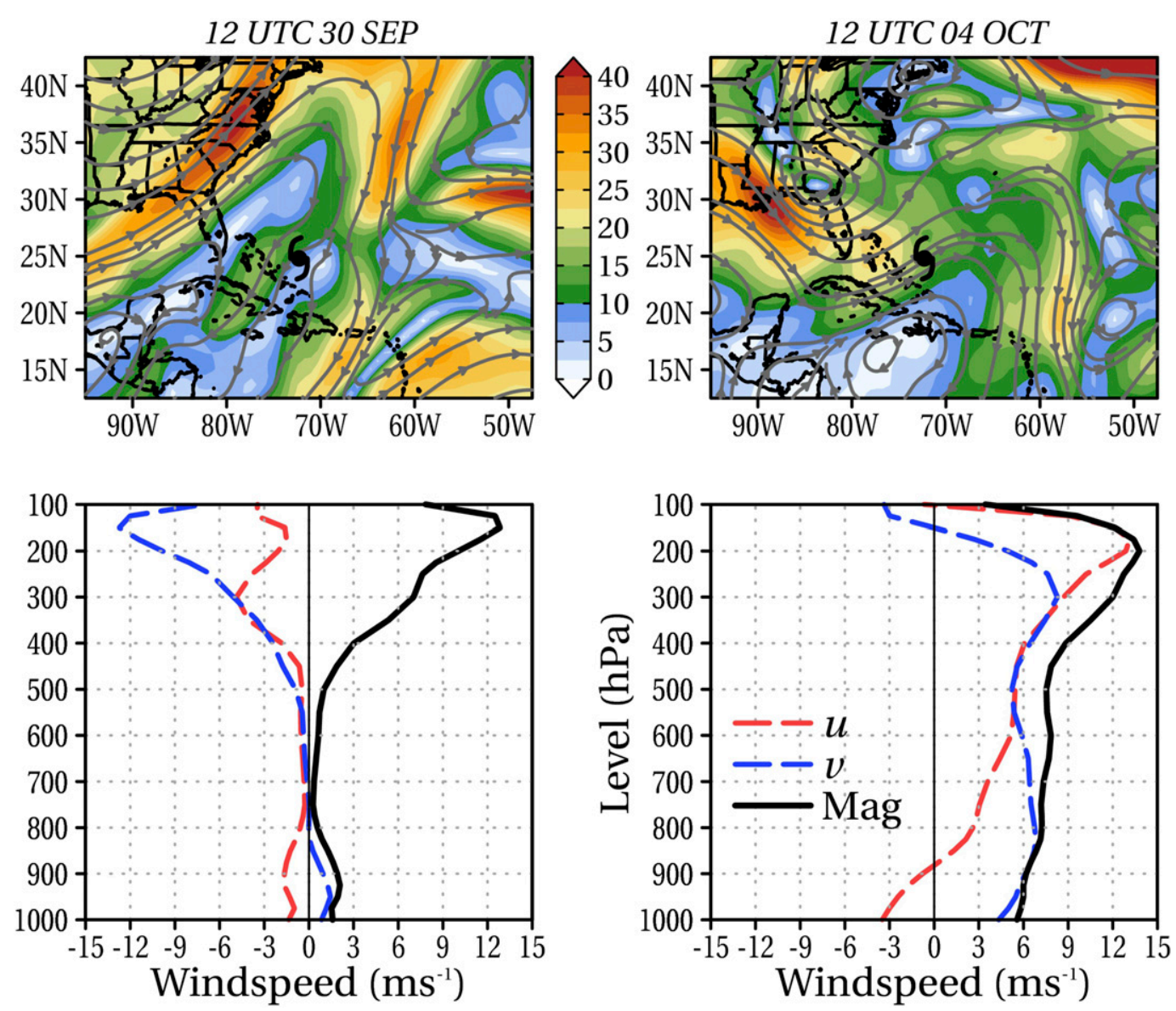

FIG. 2. (top) The 200-hPa winds $\left(\mathrm{m} \mathrm{s}^{-1}\right.$ ) around 2015 NATL Hurricane Joaquin (black TC symbol) from ERAInterim at (left) 1200 UTC 30 Sep and (right) 1200 UTC 4 Oct. (bottom) Vertical profiles of smoothed winds centered on a $5^{\circ} \times 5^{\circ}$ box around Joaquin.

illustrated in Fig. 2, the upper-level flow environment affecting Joaquin evolves from anticyclonic northeasterly flow around a ridge to southwesterly flow ahead of a transient trough passing to the north of the TC. Figure 2 also demonstrates how the wind profile changes around the storm, where the wind is first smoothed with a Shuman filter (Shuman 1957) and then averaged in a $5^{\circ} \times 5^{\circ}$ box around the storm's location. As time progresses, the depth of the environmental flow becomes greater, as opposed to the earliest times where the background flow at low and midlevels is small. The shear values, according to SHIPS, are generally similar: $10 \mathrm{~m} \mathrm{~s}^{-1}$ (see Part I). We note that the goal of this work is not meant to simulate Joaquin directly; rather, the objective here is to simulate this class of TCs from a more general perspective based on the following TCs: 2008 EPAC Hernan, 1997 EPAC Guillermo, 2015 EPAC Hilda, and so on (Part I). Additionally, analyzing a TC of this class in an idealized model setup also allows us to control for other environmental parameters such as environmental humidity and SSTs. We focus on the dynamic impacts of the shear profile only. It also must be stressed that this study will not address the phase space of these storms, which includes the sensitivity to SSTs and consideration of a broader spectrum of shear profiles. This work does not address the broader impact of TCAs on the overall evolution of the TCs. These issues will be addressed in future studies. We are mainly addressing the source of the TCAs and their structures.

\section{b. Center-finding methods}

As Ryglicki and Hart (2015) and Nguyen et al. (2014) summarized, there exist myriad ways to calculate the center of a TC, and the notion of a single "center" throughout the TC typically does not exist. Misdiagnosis of the center can result in inconsistencies when measuring tilt (Ryglicki and Hart 2015) or when decomposing 2D quantities such as vorticity or winds (Ryglicki and Hodyss 2016). Table 1 summarizes all of the methods used to calculate a center in this study. The motivation for the use 
TABLE 1. All of the center-finding methods used and their descriptions. "Class" characterizes the center-finding method per the terminology defined by Ryglicki and Hart (2015): minimization of azimuthal variance (MAV), weighted grid point (WGP), and local extreme (LE). All WGP methods use 10 iterations to convergence. See section 2 for more details.

\begin{tabular}{|c|c|c|}
\hline Abbreviation & Class & Description \\
\hline MAVWBS & MAV & $\begin{array}{l}\text { Brute force check for MMTW at each } \\
\text { grid point in } 100 \text {-km box around first } \\
\text { guess out to a radius of } 150 \mathrm{~km} \text {, then } \\
\text { Hurricane Research Division simplex } \\
\text { (Marks et al. 1992). Annulus size of } \\
4 \sqrt{ } 2 \mathrm{~km} \text { (inner domain } d x \text { multiplied } \\
\text { by } 2 \sqrt{ } 2 \text { ). }\end{array}$ \\
\hline OPI120 & WGP & $\begin{array}{l}\text { One-over-Exner-function centroid, } \\
120 \mathrm{~km} \times 120 \mathrm{~km} \text { weighting area }\end{array}$ \\
\hline OPI120-SM9 & WGP & $\begin{array}{l}\text { Same as OPI120, except for smoothed } \\
\text { Exner field }\end{array}$ \\
\hline PMIN & LE & Pressure minimum \\
\hline PMIN-SM9 & LE & $\begin{array}{l}\text { Same as PMIN, except for smoothed } \\
\text { pressure field }\end{array}$ \\
\hline PRC120 & WGP & $\begin{array}{l}\text { Pressure centroid, } 120 \mathrm{~km} \times 120 \mathrm{~km} \\
\text { weighting area }\end{array}$ \\
\hline PRC120-SM9 & WGP & $\begin{array}{l}\text { Same as PRC120, except for smoothed } \\
\text { pressure field }\end{array}$ \\
\hline PVC120 & WGP & $\begin{array}{l}\text { PV centroid, } 120 \mathrm{~km} \times 120 \mathrm{~km} \\
\text { weighting area }\end{array}$ \\
\hline PVC120-SM9 & WGP & $\begin{array}{l}\text { Same as PVC120, except for a } \\
\text { smoothed PV field }\end{array}$ \\
\hline PVC300 & WGP & $\begin{array}{l}\text { PV centroid, } 300 \mathrm{~km} \times 300 \mathrm{~km} \\
\text { weighting area }\end{array}$ \\
\hline PVC300-SM9 & WGP & $\begin{array}{l}\text { Same as PVC300, except for a } \\
\text { smoothed PV field }\end{array}$ \\
\hline PVV120 & WGP & $\begin{array}{l}\text { Blended vorticity and PV centroids, } \\
120 \mathrm{~km} \times 120 \mathrm{~km} \text { weighting box: } \\
\text { vorticity centroid up to } 500 \mathrm{~m} \text {, } \\
\text { Hermitian blend to } 4 \mathrm{~km}, \mathrm{PV} \text { centroid } \\
\text { to top of storm }\end{array}$ \\
\hline PVV120-SM9 & WGP & $\begin{array}{l}\text { Same as PVV120, except for smoothed } \\
\text { PV and vorticity fields }\end{array}$ \\
\hline VYC120 & WGP & $\begin{array}{l}\text { Vorticity centroid, } 120 \mathrm{~km} \times 120 \mathrm{~km} \\
\text { weighting area }\end{array}$ \\
\hline VYC120-SM9 & WGP & $\begin{array}{l}\text { Same as VYC120, except for smoothed } \\
\text { vorticity field }\end{array}$ \\
\hline MEANC & LE & Average of all previous centers \\
\hline
\end{tabular}

of multiple tilt calculations is that since a key component of this evolution is related to the tilt, we are able to demonstrate that our findings are robust, regardless of center-finding method. Determining statisticaldynamical relationships among the different centerfinding methods is beyond the scope of this study [see Ryglicki and Hart (2015) for more details].

For each center-finding method, the first guess is always the pressure minimum at the lowest model level. From there, an $800 \mathrm{~km} \times 800 \mathrm{~km}$ subdomain is extracted and used for calculation of the following center-finding methods. The reason for this is that during early stages of the TC, when the storm is weak, the pressure minimum is not always a reliable first guess of the center location at upper levels (Ryglicki and Hart 2015).

Many of the center-finding methods employ a Gaussianstyle nine-point smoother. This smoother is functionally identical to the smoother employed in the Grid Analysis and Display System (GrADS) as the "smth9" function. All of the spatially smoothed fields are passed through the smoother 100 times before the center-finding method is employed, similar to Stern and Nolan (2012).

Additionally, in this study, we seek to clarify the difference between "storm relative" and "tilt relative" analyses. As Ryglicki and Hodyss (2016) showed, fields that are very sensitive to center placement include fields that possess strong gradients - such as vorticity or PV for eddymean partitioning — and the radial wind. In the following sections, the data will be interpolated using three centers to cylindrical coordinates using bicubic Lagrange polynomials with 501 radial points at a spacing of $1 \mathrm{~km}$ and 360 azimuthal points at a spacing of $1^{\circ}$ (or $\pi / 180$ radians). For "storm relative" analyses, which include track and relative tilt, the low-level center is taken to be the average center of the model levels 3-9 (roughly $250 \mathrm{~m}-1 \mathrm{~km}$ ), the depth through which the background wind is constant. This also accounts for instabilities in PV centroids at the lowest model levels. Each vertical level is interpolated respective to that low-level center. For "tilt relative" analyses, each level is interpolated to cylindrical coordinates around its own respective center. The three centers that are used for interpolation are a tilt-relative wind method, which seeks to maximize the mean tangential wind at the radius of maximum winds (RMW) (MAVWBS); a tilt-relative PV-vorticity hybrid centroid (PVV120); and stormrelative (surface centered) minimum of a smoothed pressure field (PMIN-SM9). These three methods provide a way to test the robustness of a physical feature.

\section{General diagnostics}

Since we have chosen to use an idealized model, a "verification" in a strict sense-such as a direct comparison of a model forecast with observational data (e.g., Smith et al. 2017)—cannot be readily performed. With that in mind, this section describes basic features of the simulations including the intensity, the general similarities between synthetic satellite imagery of the CM1 and satellite observations, and perhaps most importantly, the tilt of the vortex. Understanding the behavior of the tilt of the vortex is critical to the entire intensification process; therefore, a thorough diagnostic evaluation is undertaken.

\section{a. Intensity}

Figure 3 shows the minimum pressure, maximum mean tangential wind (MMTW), and RMW, all at the 

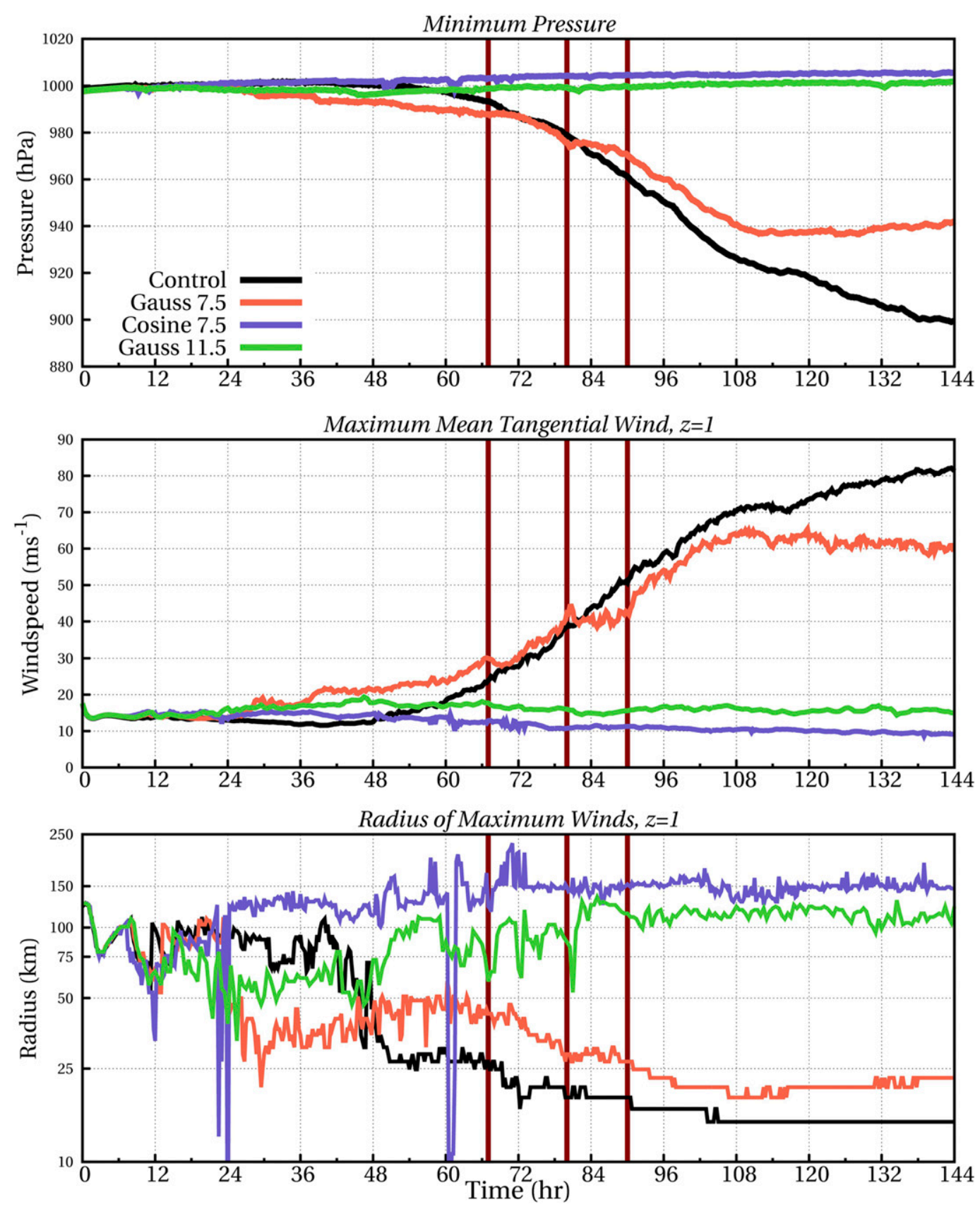

FIG. 3. (top) Minimum pressure, (middle) MMTW, and (bottom) RMW, all at the lowest scalar model level, for the four simulations. Dark red lines correspond to the first RI period, the levelling off of intensification, and the second abrupt RI.

lowest scalar model level $(50 \mathrm{~m})$, for the four simulations. The maximum mean tangential wind and the RMW are taken from the wind maximum center-finding method. It should be noted that the evolutions of the sheared simulations are closer to dry vortices at the earliest model times (Jones 1995, 2000; Smith et al. 2000) due to the lack of an initial secondary circulation and diabatic effects. For reference, the first appearance of convective activity within the vortex in all simulations occurs $8 \mathrm{~h}$ into the simulation. Subsequently, there is a stark contrast in the evolution of the three sheared vortices. In the cosine-shear simulation and in the stronger $11.5 \mathrm{~m} \mathrm{~s}^{-1}$ Gaussian-shear simulation, the TC does not intensify at all. In contrast, in the $7.5 \mathrm{~m} \mathrm{~s}^{-1}$ Gaussian-shear simulation, the TC does intensify, and the behavior is quite different. 
The $7.5 \mathrm{~m} \mathrm{~s}^{-1}$ Gaussian-shear simulation goes through several stages on its path to RI. Deviation from the control begins $24 \mathrm{~h}$ into the simulation. This is followed by 3.5 days of steady intensification. A briefer (or a stronger) intensification period, with an increase in intensity of $15 \mathrm{~m} \mathrm{~s}^{-1}$ in $13 \mathrm{~h}$, occurs from 67 to $80 \mathrm{~h}$. Prior to this period, the RMW expands. During this period, the RMW contracts from 47 to $28 \mathrm{~km}$. After that, from 80 to $90 \mathrm{~h}$, the low-level intensification temporarily ceases. An abrupt second intensification occurs between 90 and $108 \mathrm{~h}$. During this time, the RMW contracts again from 28 to $18 \mathrm{~km}$. At $108 \mathrm{~h}$, the intensity trace indicates that the TC has peaked in terms of intensity, and the TC undergoes a very slow weakening thereafter. These multiple stages of intensification are in contrast to the control simulation. In the control simulation, the TC begins its intensification around $40 \mathrm{~h}$ and intensifies at a steady rate until $114 \mathrm{~h}$ into the simulation, where it pauses at $70 \mathrm{~ms}^{-1}$ for $9 \mathrm{~h}$ [this is due to a low-level vorticity rearrangement (not shown)] before it resumes its intensification process and reaches an intensity of $81 \mathrm{~m} \mathrm{~s}^{-1}$ at the end of 6 days. It is also important that we mention at this point that motivated by the results of Tao and Zhang (2015) in terms of intrinsic predictability of TCs in shear, we performed sensitivity tests by varying the shear magnitude $\pm 0.01 \mathrm{~m} \mathrm{~s}^{-1}$. The results are robust: the $7.5 \mathrm{~m} \mathrm{~s}^{-1}$ Gaussian-shear TCs develop, and the cosine-shear TCs do not.

\section{b. Synthetic satellite imagery}

Per recommendations from the documentation of the CM1 (http://www2.mmm.ucar.edu/people/bryan/ $\mathrm{cm} 1 /$ README.namelist), the more realistic radiation code available internally to CM1r18 was not used, in part for simplicity to remove any diurnal forcing and since the current domain, roughly $6000 \mathrm{~km} \times 4000 \mathrm{~km}$, is very large. In lieu of a diurnally varying radiation forcing, the simulations were carried out with Newtonian cooling capped at $2 \mathrm{~K} \mathrm{day}^{-1}$. To calculate the respective simulated brightness temperatures-both IR and WVthe CM1 output was passed to the Community Radiative Transfer Model (CRTM; Van Delst 2013; Grasso et al. 2008; Bikos et al. 2012; Jin et al. 2014). Given the relatively unrealistic clear-sky radiation properties of the idealized simulation, we focus on qualitative similarities between satellite observations and the model results. The explicit documentation of the slow migration of the convection and the periodicity (see Figs. 12 and 14 from Part I) is performed using model-derived convection, as described in section 4. Figure 4 shows the IR imagery of Hernan, Norbert, Fabio, and Hilda, along with the synthetic satellite imagery from the $7.5 \mathrm{~m} \mathrm{~s}^{-1}$ Gaussian-shear simulation and the control simulation. Qualitatively, the convective feature in Fig. 4e matches the shape, temperature, and shear-relative orientation of the observations. In the control simulation (Fig. 4f), convection is never as localized as it is in the other simulations.

\section{c. Tilt}

A key part of the evolution of the TCs in this manuscript is the migration of the vortex tilt. Figure 5 shows the evolution in time of the storm-relative tilt of the developing vortex, smoothed with a 2.5 -h running mean (to remove transients) and analyzed using five different center-finding methods. We have included at least one method from each class defined by Ryglicki and Hart (2015): local extreme, weighted grid point, and minimization of azimuthal variance. The fact that the tilt grows at early lead times is expected and consistent with other work (e.g., Frank and Ritchie 2001; Stern and Zhang 2013). A key part of the simulation occurs from 60 to $90 \mathrm{~h}$. This period covers the first period of intensification and the levelling off of intensification apparent in Fig. 1. According to nearly all of the centerfinding methods, the upper levels $(9,11 \mathrm{~km})$ and midlevels $(6,7 \mathrm{~km})$ begin to wobble out of phase. This can be seen by the interweaving of the upper-level tilt magnitudes $(11,9 \mathrm{~km})$ and the midlevel tilt magnitudes in the PV centroids (Figs. 5d,e) or the evolution of the tilt magnitudes in opposite directions in the pressure minimum (Fig. 5c) starting at $60 \mathrm{~h}$ and ending in the 84-90-h time frame. At $84 \mathrm{~h}$, the oscillations cease, and the midlevel tilts (6- and 7-km centers) slowly begin to decrease. The second rapid intensification phase begins at $90 \mathrm{~h}$, as several center-finding methods indicate that the lower and midlevels of the simulated TC are now vertically aligned.

Figure 6 a further elucidates the evolution of the 6-km tilt, a key part of this evolution examined more thoroughly in section 4, as analyzed by the PV-vorticity hybrid center for the first $84 \mathrm{~h}$ of the simulation. Over the first $24 \mathrm{~h}$, the tilt reaches its maximum extent, primarily downshear left. Shortly thereafter, the TC slowly begins the realignment process. It is not clear whether this is due to induced flow from the surface (Jones 1995) or due to a resonant damping mechanism (Schecter et al. 2002; Reasor et al. 2004). Nevertheless, a higher-order nutation in the tilt precession is evident, as the tilt lingers in one general area for several hours before swinging counterclockwise. A "nutation" is defined as a periodic variation in the inclination of the axis of a rotating object. At $48 \mathrm{~h}$ into the simulation, the tilt migrates into a downshear-left position, conceptually agreeing with idealized theory (Reasor et al. 2004; Reasor and Montgomery 2015). Eventually, between 72 and $84 \mathrm{~h}$, the midlevel center orbits a left-of-shear axis. This is the 
(a) Hernan, 1830 UTC 07 Aug

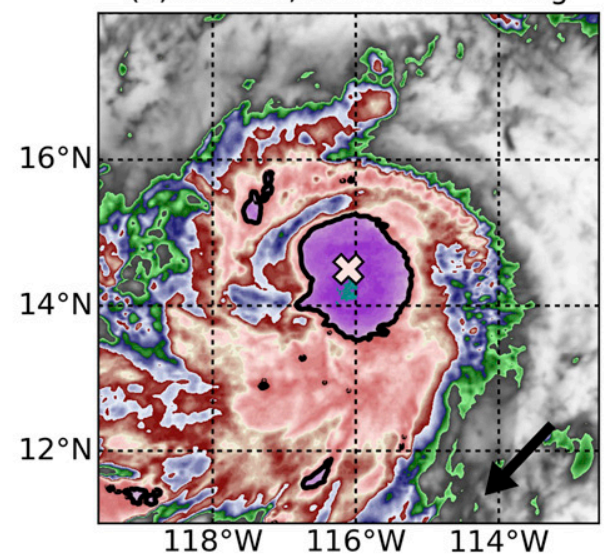

(c) Fabio, 1800 UTC 13 Jul

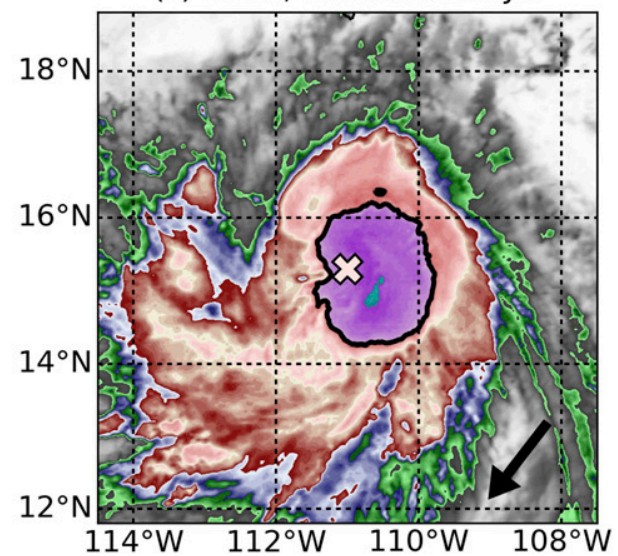

(e) CM1-shear, $65 \mathrm{hr} 45 \mathrm{~min}$

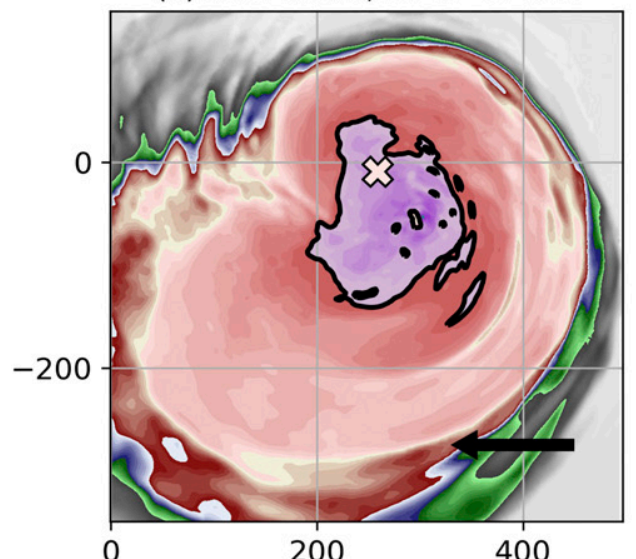

0 (b) Norbert, 0245 UTC 07 Oct

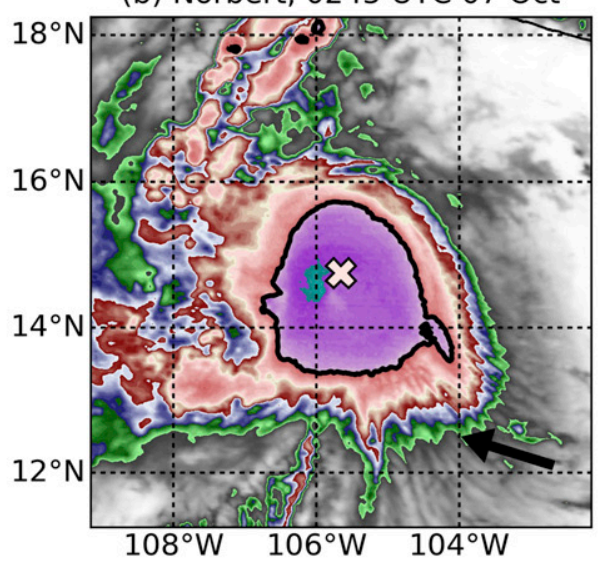

(d) Hilda, 0500 UTC 07 Aug

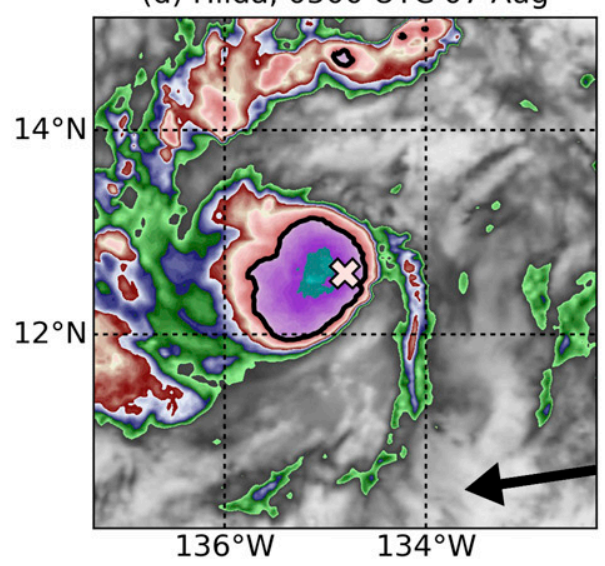

(f) CM1-control, $65 \mathrm{hr} 45 \mathrm{~min}$

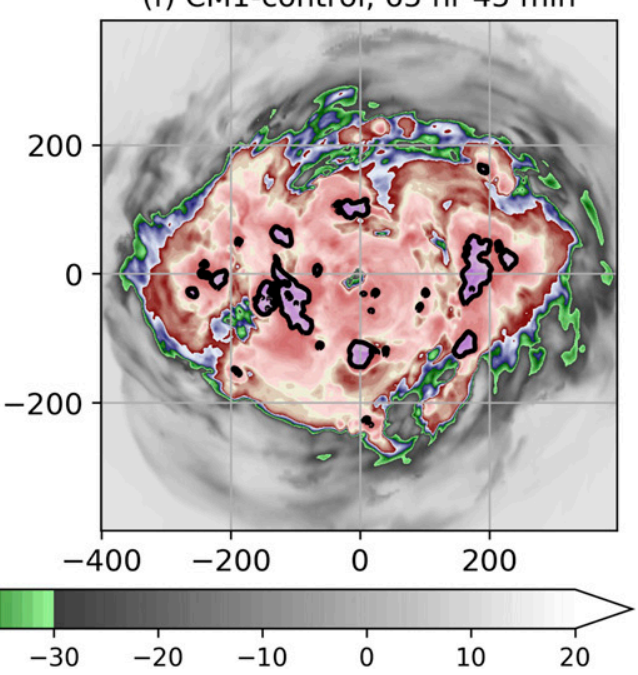

FIG. 4. Infrared satellite imagery $\left({ }^{\circ} \mathrm{C}\right)$ for (a) 2008 Hernan, (b) 2008 Norbert, (c) 2012 Fabio, (d) 2015 Hilda, (e) the sheared CM1 simulation (synthetic), and (f) the control CM1 simulation (synthetic). Black arrows are shear direction only. Units of the abscissa and ordinates of the CM1 simulations are $\mathrm{km}$. Crisscross is either the interpolated (a)-(d) best track location or (e) low-level center. 

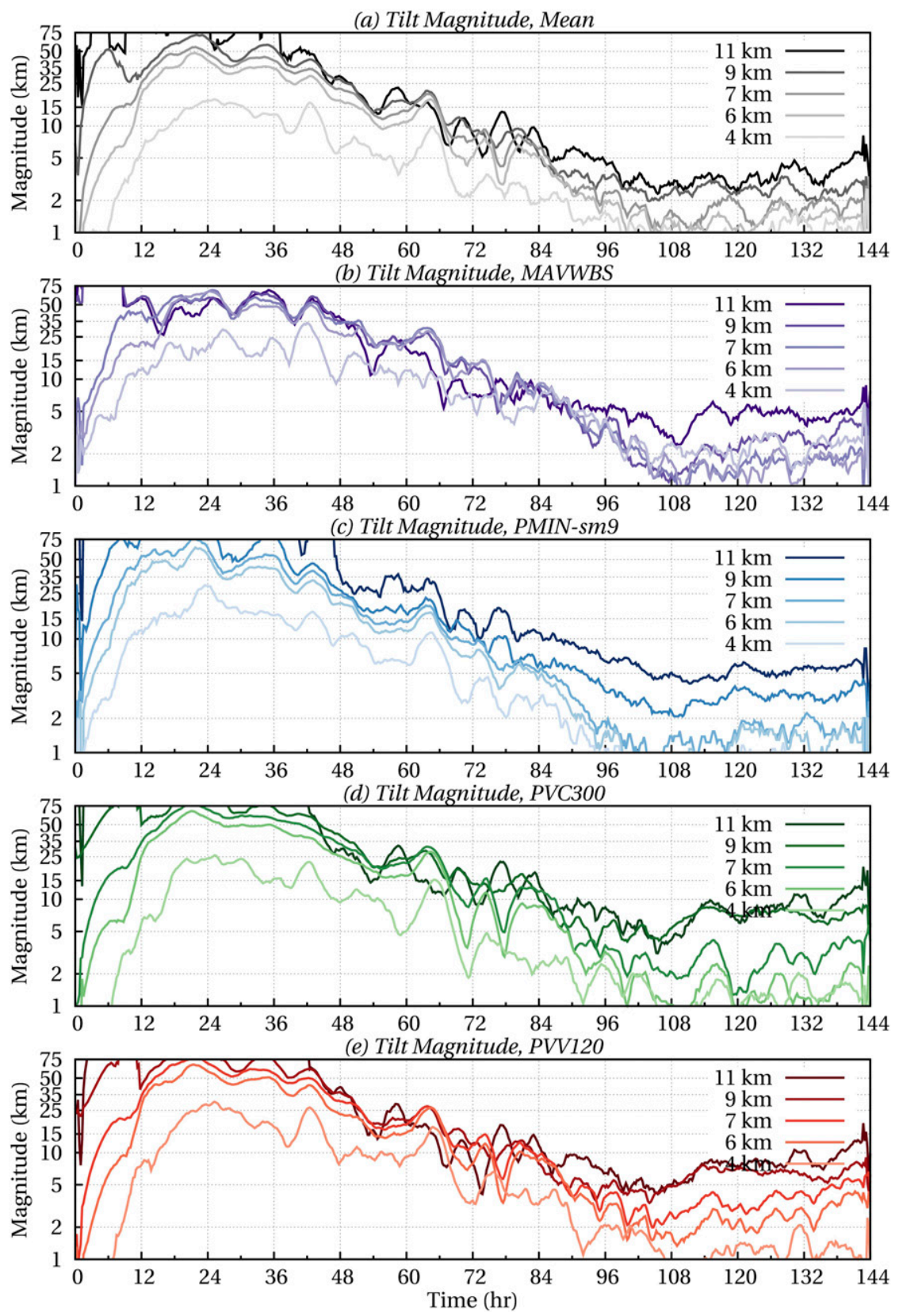

FIG. 5. Time series of the storm-relative tilt, smoothed with a 2.5-h running mean, at 4-, 6-, 7-, 9-, and 11-km heights for five different center-finding methods: (a) MEANC, (b) MAVWBS, (c) PMIN-SM9, (d) PVC300, and (e) PVV120. See Table 1 for acronyms.

two-dimensional display of what was shown in the tilt magnitudes and their oscillations in the middle of the simulation (see Fig. 5). As an additional note, the 6-km center of the cosine-shear TC never returns upshear (Fig. 6b), implying that the induced flows from the two levels are unable to interact with each other strongly enough to overcome the environmental flow, which agrees qualitatively with previous studies (Jones 1995; Ge et al. 2013; Zhang and Tao 2013; Tao and Zhang 2015;
Finocchio et al. 2016). After approximately $36 \mathrm{~h}$, the center-finding calculation becomes erratic and unstable as the TC fails to develop vertically; the other center-finding methods behave in similar ways. The Gaussian-11.5 TC additionally exhibits similar characteristics (not shown).

A basic analogy for the tilt evolution is that of a gyroscope or a spinning top. Imagine a perfectly spinning, frictionless symmetric top, where the axis of rotation is 

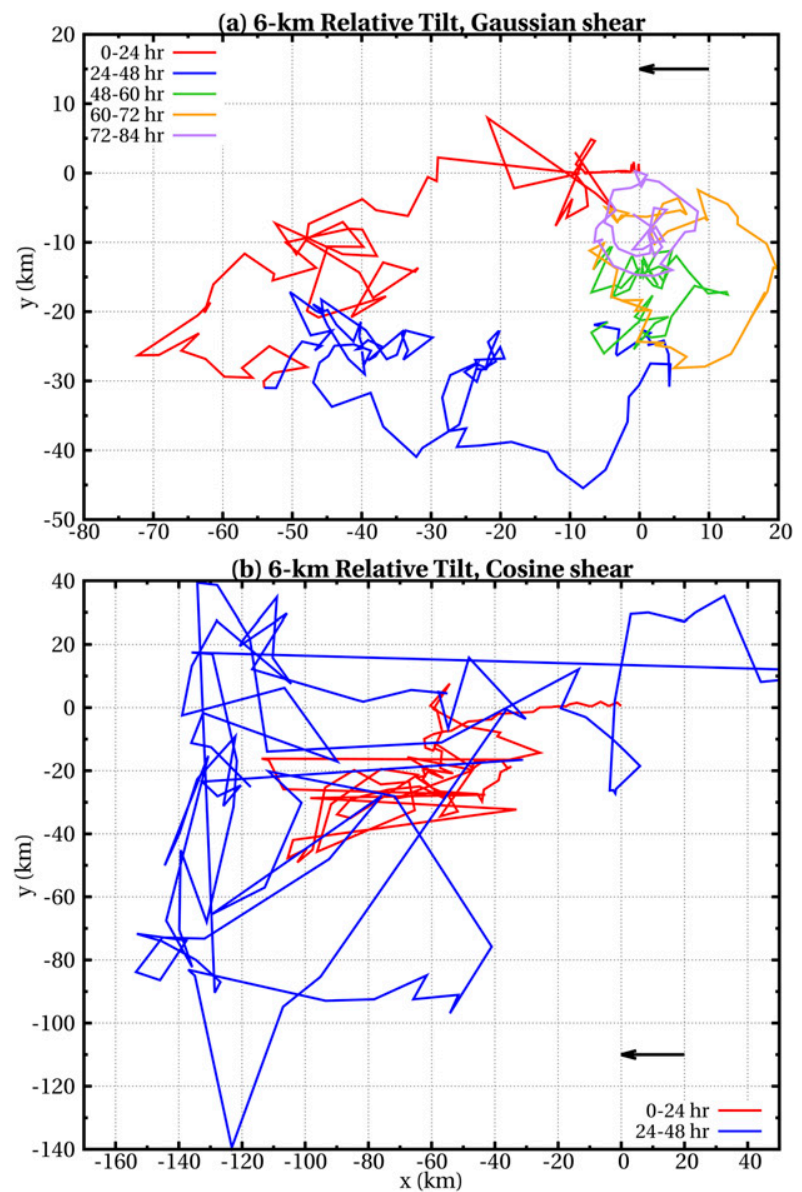

FIG. 6. Location of the 6-km center normalized by the low-level center using the PVV120 method (see Table 1), color coded by simulation time, for the (a) $7.5 \mathrm{~m} \mathrm{~s}^{-1}$ Gaussian-shear TC and (b) cosine-shear TC. Note changes in ordinate and abscissa. Black arrows are shear vectors.

aligned with the vertical axis. When displaced from the vertical axis (i.e., tilted), the top begins precessing around the vertical axis as a result of the torque exerted by gravity. If the initial displacement occurs sufficiently slowly (negligible acceleration), then the top precesses at a constant rate at a given tilt angle. If the displacement occurs quickly, then the top will overshoot the angle, and a higher-order nutation (or wobble) will develop around the axis of rotation. Relating this concept to that of the developing TC here, the precession is the long, slow realignment, and nutations are the higher-order deviations from that precession. Using a similar lowpass windowed-sinc filter from Part I, Fig. 7a illustrates the low-pass (precession) and the high-pass (nutation) evolutions of the tilt. Using this methodology, the nutations on the precession are responsible for approximately $10-20-\mathrm{km}$ variations in the tilt magnitude, and these nutations exist almost immediately. This also has
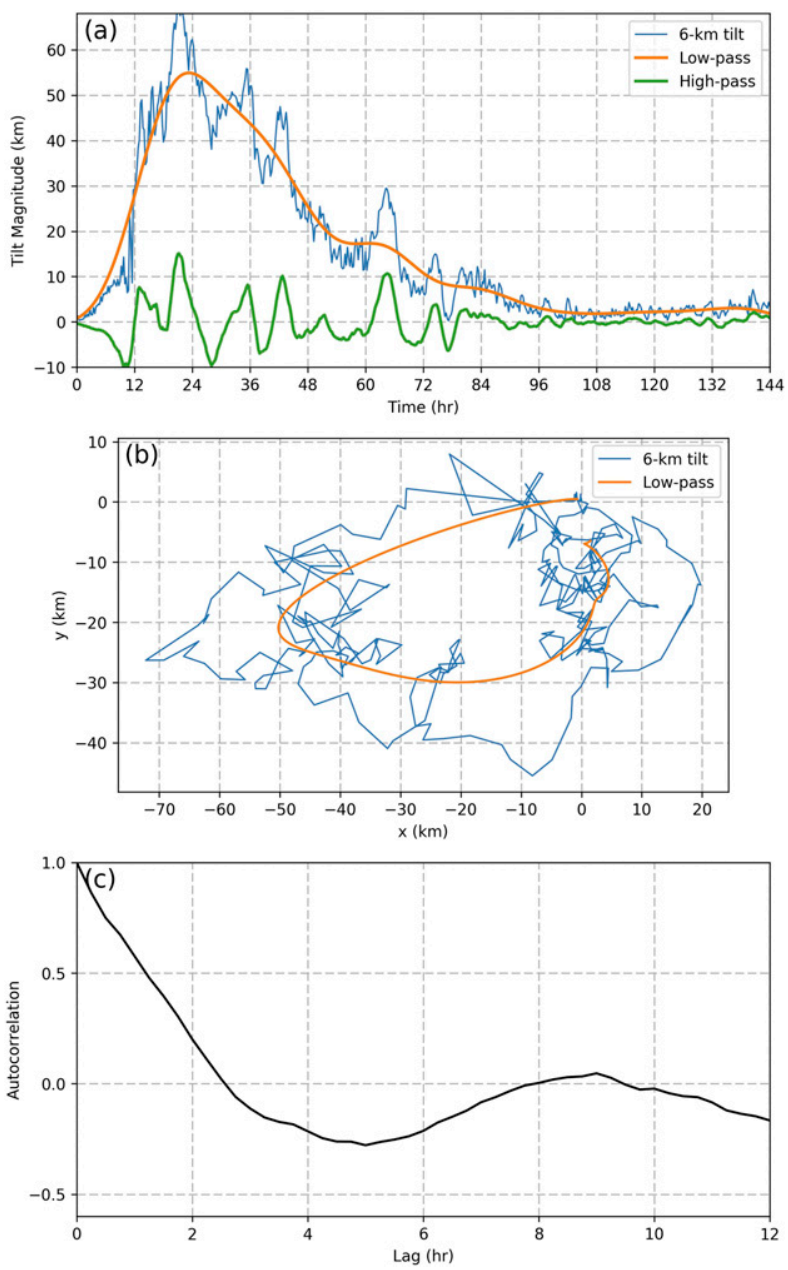

FIG. 7. (a) The 6-km tilt magnitude (blue line) filtered with a lowpass filter (orange line) in order to extract the high-pass signal (green line). Orange line signifies the precession; green line signifies the nutations. The green line is smoothed with a 2-h running mean to eliminate spurious noise associated with center finding. (b) Spatial evolution of the 6-km tilt (blue line) and its low-passfiltered evolution (orange line) up to $84 \mathrm{~h}$. (c) The autocorrelation of the high-pass time series.

the consequence of changing the spatial evolution of the tilt as well (Fig. 7b), as the nutation rotations are superimposed on the tilt precession.

Finally, when performing an autocorrelation analysis on the nutation tilt magnitude (Fig. 7c), as was done for the cloud cover in Part I after the diurnal component was removed, the largest anticorrelation is $5 \mathrm{~h}$, which indicates a nutation of $9-10 \mathrm{~h}$. While this is slightly longer than the 4-8-h window from Part I, it is well within reason in order to explain the periodicity observed (we explore this further in section 5). It is also worth reiterating our discussion from the appendix in Part I that the autocorrelation function, while simpler to interpret than a wavelet, operates in the frequency 
domain only, so it is not suited for determining localized details in a time series as short as this one. This is especially true as the nutation magnitude becomes essentially zero halfway through the simulation; however, it can provide a simple analysis of the dominant temporal mode, which is how it is used here.

\section{Tilt-modulated convective asymmetry structure}

This section focuses on both the mesoscale characteristics of the TCA (and the tilt) and the smaller-scale characteristics of individual convective towers within them. A key feature here is that convection and the tilt are very closely related. The tilting of the vortex changes the thermal structure of the vortex, and this alters the characteristics of the individual towers rising within the TCA when compared with those in the control simulation. Perhaps the most important fact is that the towers in the sheared simulation are thermally buoyant, and this appears to alter the vorticity structure of the tower in the vertical. This section discusses both.

\section{a. TCA characteristics}

The primary interplay in the evolution of the simulated TC in Gaussian shear before the second RI period is the one between the vortex tilt and precipitation (convection). To quantify this relationship, the total condensed water of the column (TCWC) is examined. Total condensed water of the column is a two-dimensional quantity defined as

$$
\mathrm{TCWC}=\int_{0}^{z_{\mathrm{top}}} \rho\left(r_{c}+r_{r}+r_{i}+r_{s}+r_{g}\right) d z
$$

where $\rho$ is the dry atmospheric density, and $r$ is a mixing ratio of cloud water $c$, rainwater $r$, ice $i$, snow $s$, or graupel $g$, the last of which is the "large ice" specie in the chosen microphysics scheme. This is a generalization that includes both liquid water path and ice water path, a factor that allows this metric to indicate deep convection better than reflectivity since it accounts for a total vertical integral of all water condensate (e.g., $\mathrm{Xu}$ and Randall 2001; Carrió et al. 2005; Pattnaik et al. 2011). Figure $8 \mathrm{a}$ is a radial sum of total condensed water of the column at each azimuth and demonstrates that this field for the Gaussian-shear storm is decidedly asymmetric over the first $96 \mathrm{~h}$ of the simulation, especially when compared with its nonsheared control counterpart (Fig. 8b). While convection is asymmetric, as expected with a sheared TC (e.g., Frank and Ritchie 2001; Reasor et al. 2009, 2013), there is additional complexity to this evolution. The first major convective event in the simulation, as evidenced by localized high values of total
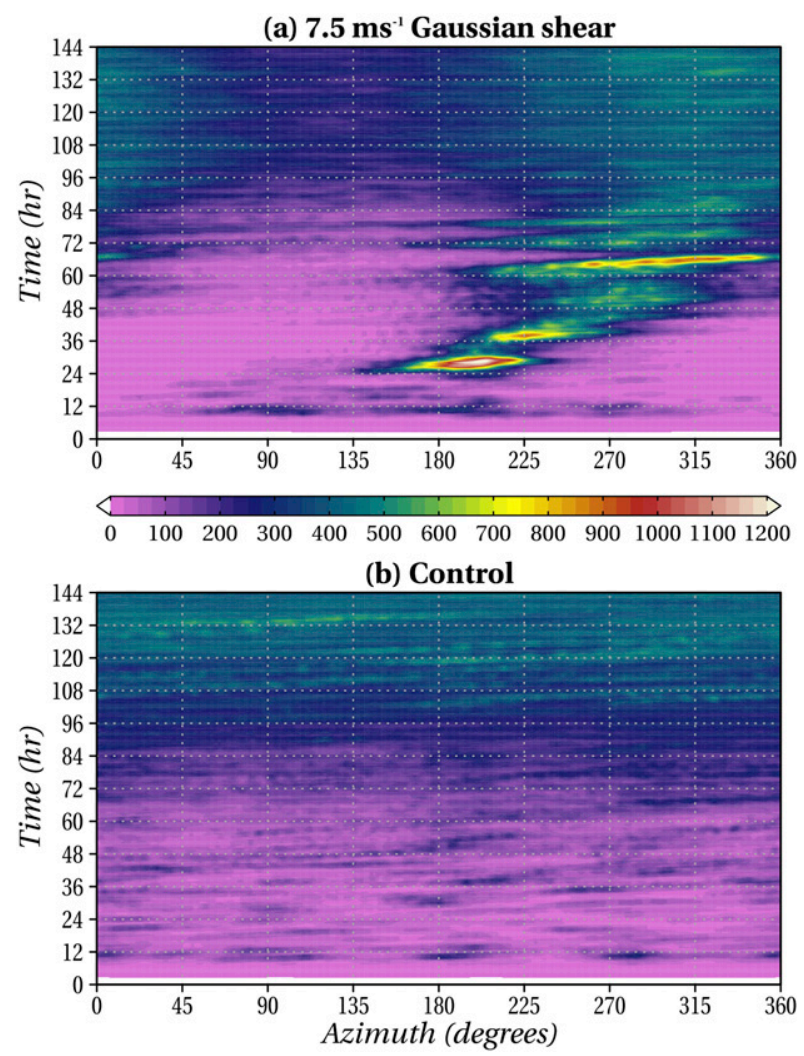

FIG. 8. Storm-relative TCWC $\left(\mathrm{kg} \mathrm{m}^{-2}\right)$, radially summed from 0 to $100 \mathrm{~km}$, for the (a) Gaussian-sheared vortex and (b) control vortex at each azimuth, smoothed with a 2.5 -h running mean. Azimuth angles are math convention, such that $0^{\circ}$ is east (upshear), $90^{\circ}$ is north (right of shear), $180^{\circ}$ is west (downshear), and $270^{\circ}$ is south (left of shear).

integrated condensate, occurs at $27 \mathrm{~h}$ and is located in the downshear-left quadrant of the simulated TC. The broader convective maximum begins a slow migration over the next $24 \mathrm{~h}$ to left of shear and upshear left. Beginning at approximately $60 \mathrm{~h}$, three readily identifiable streaks of large values of total condensed water of the column propagate from downshear left to almost completely upshear with gaps in between. These streaks signify the TCAs. After $90 \mathrm{~h}$, the storm becomes more symmetric, which is also the same time that the second RI period takes place (Fig. 3).

One notable aspect regarding the movement of these integrated condensate streaks is their relative slow translation speed-especially compared with the mean flow-around the vortex. Given the general characteristics of the maximum mean tangential wind as $30 \mathrm{~m} \mathrm{~s}^{-1}$ and the RMW as $45 \mathrm{~km}$ at $60 \mathrm{~h}$ (see Fig. 3), an air parcel will circumnavigate the eyewall in $2.62 \mathrm{~h}$. The first of these TCAs takes approximately $7 \mathrm{~h}$ to move around only a third of the vortex. There is only one feature of this vortex that is moving that slowly: the tilt. However, 
there is more nuance to this explanation than simply "the tilt," as the tilt does not consistently point in one direction with height (Fig. 5). Figure 9 is a snapshot of some of the tilt calculations and total condensed water of the column at $65 \mathrm{~h}, 30 \mathrm{~min}$ into the simulation in the middle of the first TCA. As a reminder, definitions of the tilt acronyms are present in Table 1 . Every centerfinding method presented here is oriented upshear left up to $6 \mathrm{~km}$ and then bends back downshear above that. While one could theoretically argue that PV centroids are naturally biased toward convection at midlevels, this does not explain similar behavior from wind-based centers, pressure minima, or the overall mean of the centers. Moreover, as discussed by Ryglicki and Hart (2015), PV centroids with large weighting boxes are designed to be more resistant to spuriously high values of PV associated with convection and to capture the larger-scale tilt of the vortex.

Figure 8a is reproduced in Fig. 10, but with tilt considerations. The left panel in Fig. 10 shows that the convection migrates upshear with the 6-km tilt angle. A careful analysis indicates that the convection and the midlevel center, simply defined here as the 6-km center, are coincident with each other, and the convection moves with the precession of the 6-km center regardless of the tilt magnitude (Fig. 10, right panel). The majority of the local maxima in convection are associated with counterclockwise movements on smaller time scales than the multiday realignment precession. The TCAs are separate from the overall realignment precession by the $90^{\circ}$ swings, starting at $60 \mathrm{~h}$, within the span of $6-8 \mathrm{~h}$. Using the assumption that underlying convection is directly related to cold cloud temperatures (see section $3 b$ ), we can relate this evolution to observations (Part I). The slow migration of the coldest cloud tops seen in Part I over the span of $24-48 \mathrm{~h}$ is actually the convection associated with the precession of the midlevel tilt. The 4-8-h TCAs, as seen in satellite, are associated with the higher-order nutations of the tilt when the midlevel tilt precession reaches left of shear to upshear left. Based on Fig. 7a, the TCAs, as observed in satellite, occur when the precession location is $90^{\circ}$ left of shear and the precession magnitude is approximately equivalent to a full nutation magnitude-in this case, $15 \mathrm{~km}$.

The primary driver of convective initiation in initial TC evolution is thought to be primarily in the boundary layer: vorticity aggregation in the rotating convective, or "vortical hot tower," paradigm (Hendricks et al. 2004; Montgomery et al. 2009). Figure 11 is a snapshot of total condensed water of the column, $\theta_{e}$, vertical vorticity, and winds at the lowest model level at the same time of Fig. 9. The highest values of vorticity and winds are downwind of (i.e., counterclockwise from) the total

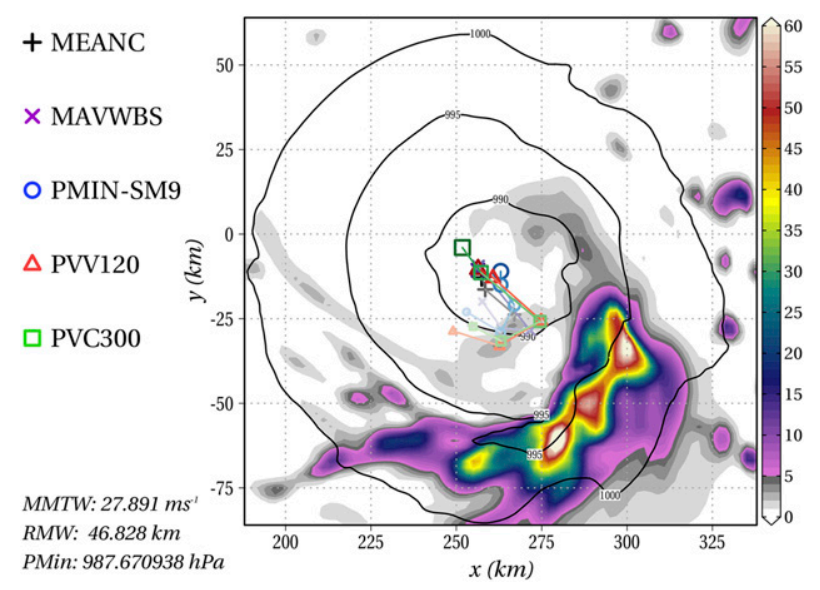

FIG. 9. TCWC $\left(\mathrm{kg} \mathrm{m}^{-2}\right)$, lowest model level pressure (hPa), and five center-finding methods (see Table 1 ) at $65 \mathrm{~h}, 30 \mathrm{~min}$ of the Gaussian-shear TC. For the center-finding methods, the heights are $1,3,6,9$, and $11 \mathrm{~km}$. The larger, darker marks are $1 \mathrm{~km}$, with decreasing size and lighter colors as heights increase.

condensate maximum. Vorticity cells upwind of (i.e., clockwise from) the TCA are not coupled with any deep convection. Shortly afterward, after the tilt reaches a relative minimum in magnitude, the deep convection corresponds fairly well with low-level vorticity on all sides of the TC near the RMW (Fig. 12). Note that even though the maximum local winds for each snapshot are different $\left(43 \mathrm{~m} \mathrm{~s}^{-1}\right.$ in Fig. 11; $33 \mathrm{~m} \mathrm{~s}^{-1}$ in Fig. 12), the maximum mean tangential wind at the two times is virtually the same.

One key forcing mechanism for the TCA is due to a decrease in the static stability in the vertical due to the tilt. In a tilted TC, a thermally wind-balanced temperature anomaly must exist due to the changes in local vortex wind shear with height (Jones 1995; DeMaria 1996). In height coordinates, the thermal wind equation for a vortex in gradient wind balance is (Hodyss and Nolan 2008)

$$
\xi \frac{\partial v}{\partial z}-\frac{N^{2}}{g}\left(\frac{v^{2}}{r}+f v\right)=\frac{g}{\theta_{\rho}} \frac{\partial \theta_{\rho}}{\partial r},
$$

where $v$ is the tangential velocity, $\xi$ is the modified Coriolis parameter, $f+2 v / r, N^{2}$ is the static stability, $g$ is gravity, $f$ is Coriolis, and $\theta_{\rho}$ is density potential temperature (DPT; see appendix A). As the vortex tilts, the local vertical wind shear increases downtilt, so the radial thermal gradient becomes more negative (i.e., colder). The second term on the left of Eq. (4) is usually small since static stability in the core is small. In a tilt-relative framework, this dipole very clearly exists, as shown in Fig. 13a.

Several key features are prominent in Fig. 13. First, it should be stated that this thermal dipole exists very early in the simulation before the primary convection begins 
Summed TCWC $\left(\mathrm{kg} \mathrm{m}^{-2}\right)$

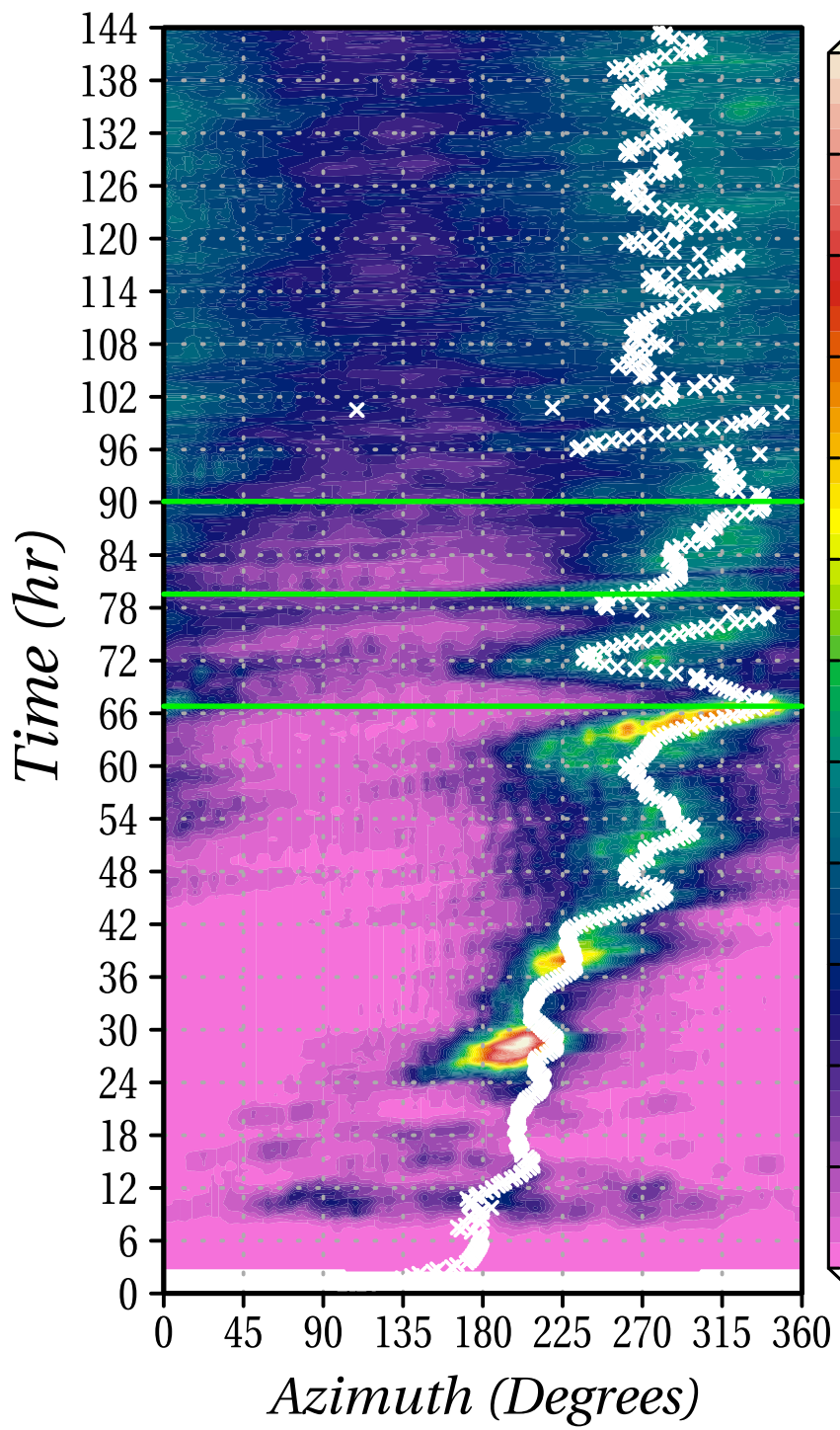

6-km Tilt, RMW

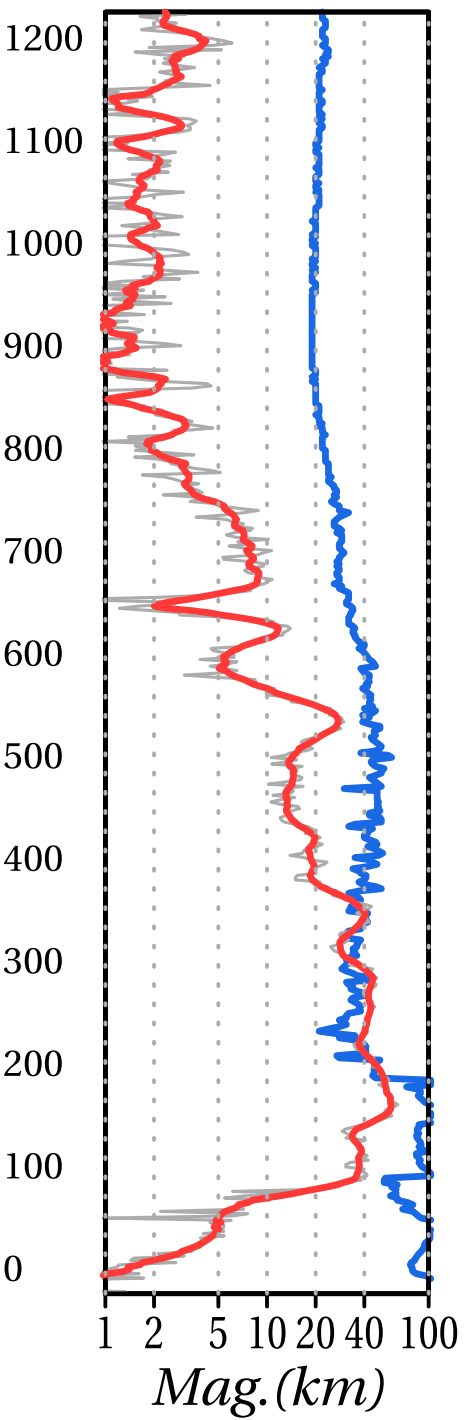

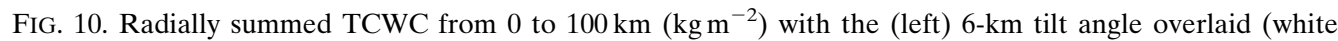
crisscrosses) and (right) 6-km tilt (red line) and RMW (blue line), where the tilt is calculated using the hybrid vorticity-PV center. All fields are smoothed with a 2.5-h running mean, except the gray line, which is the unsmoothed tilt magnitude, and the RMW (blue line). Green lines indicate first RI period, leveling off of intensification, and second abrupt intensification.

in earnest. Jones (1995) showed that due to this thermal anomaly, there is an adiabatic reactionary vertical velocity pattern, with upward motion to the right of the tilt and downward motion to the left of the tilt; however, as Frank and Ritchie $(1999,2001)$ showed, and as demonstrated here (Figs. 13a,b), in the presence of moisture, vertical motion becomes collocated with the cold anomaly. Explicitly, the vertical mass flux maximum is coincident with the total condensed water of the column maximum, and the cold anomaly is associated with the tilt of the vortex.
Deducing the structure of the cold anomaly in the vertical is more challenging because the vortex is tilted. Comparing a downtilt tilt-relative temperature anomaly with that of a storm-relative anomaly (Figs. 13c,d) shows some significant differences above 7-km height. This is a computational artifact of a misplaced center [see Ryglicki and Hodyss (2016) for more discussion], since the warm core associated with the vortex is not directly over the surface center at all heights. This is an artifact of a storm-relative coordinate system. When accounting 


\section{Time: 065 hr $30 \mathrm{~min}$ MMTW: $27.891 \mathrm{~ms}^{-1} \quad$ RMW: $46.828 \mathrm{~km}$}

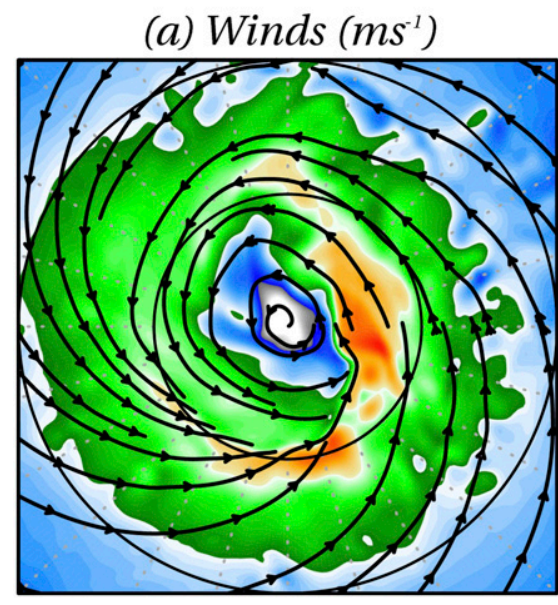

(c) Eq. Pot. Temp. (K)

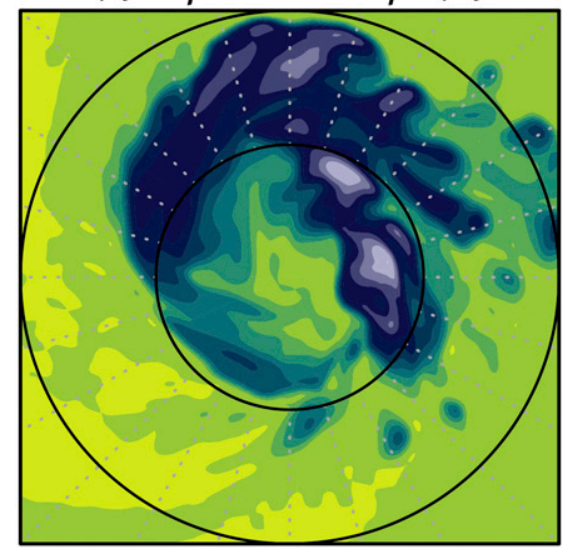

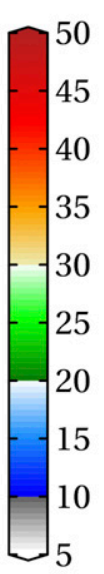
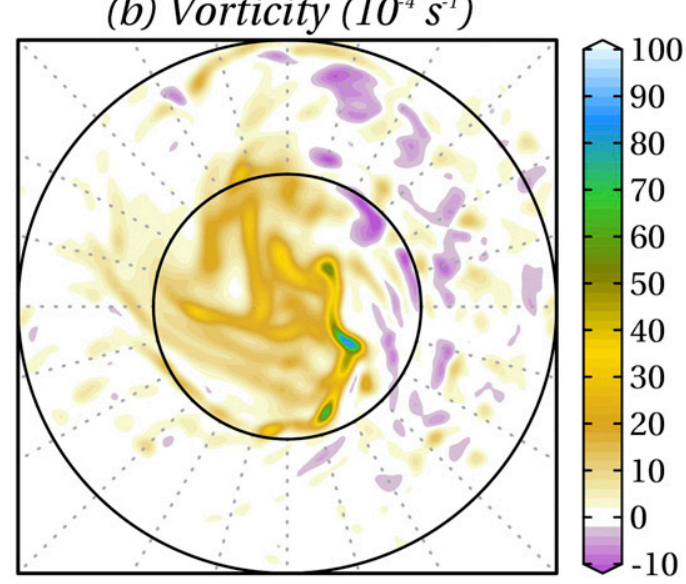

(d) TCWC $\left(\mathrm{kg} \mathrm{m}^{-2}\right)$

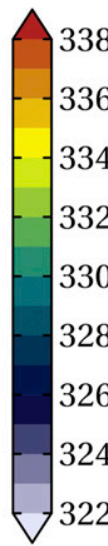

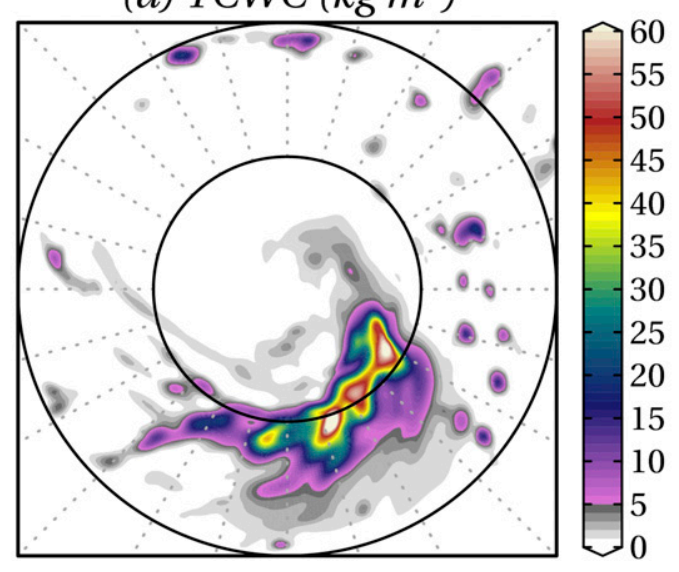

FIG. 11. Snapshot at 65 h, 30 min of the Gaussian-sheared TC's (a) winds, (b) vorticity, (c) equivalent potential temperature, all at the lowest model level, and (d) TCWC. MMTW and RMW are displayed at top. Black circles are radii at every $50 \mathrm{~km}$.

for this displacement in a tilt-relative sense, the cold anomaly appears above $7 \mathrm{~km}$. Additionally, as we show in the next subsection, individual cells that are rising underneath the tilt are thermally buoyant all the way to the top of the TC.

\section{b. Individual cells within a TCA}

Figure 14 is a collection of five azimuthal cross sections on the southern, or left of shear, side of the storm through one of these TCAs from 65 to $66 \mathrm{~h}$ of the simulation averaged from $45-$ to $55-\mathrm{km}$ radius. Small cells confined to the boundary layer start upwind in the boundary layer. As these cells progress through this TCA, they begin to grow in the vertical. At the lowest model level, the maximum mean tangential wind at this time is $28 \mathrm{~m} \mathrm{~s}^{-1}$. Assuming that the azimuthal advection of the cells is driven primarily by the mean circulation, these cells at low levels in this radial band should move $28^{\circ}$ in 15 min (i.e., per output time), which is faster than the TCA itself. The TCA is a large, slow packet of aggregate convection comprising faster-moving smaller, individual convective cells. As these cells move through this TCA, their structure begins with a local vorticity maximum coupled with vertical velocity out of the boundary layer. In the 6-8-km layer, a second vorticity maximum exists in all individual towers. This secondary vorticity maximum is persistent and very prominent in the strongest updraft considered here. For example, we highlight the updraft at $290^{\circ}$ at $65 \mathrm{~h}, 30 \mathrm{~min}$ into the simulation in Fig. 14c.

The vertical structures of the individual updrafts inside the TCA seem to be unique when compared with previous studies. Moon and Nolan (2015) provided an extensive study of the four kinds of rainbands: primary, secondary, distant, and inner. In the control simulation here, three convective maxima appeared along a 


\section{Time: 069 hr 15 min MMTW: $27.838 \mathrm{~ms}^{-1} \quad$ RMW: 42.828 km}

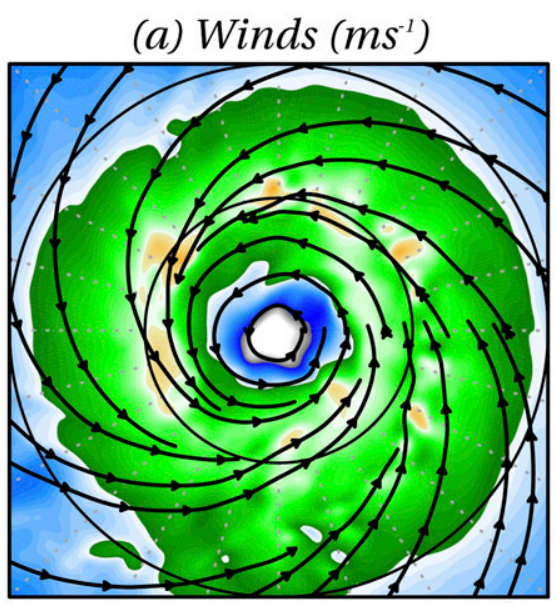

(c) Eq. Pot. Temp. (K)

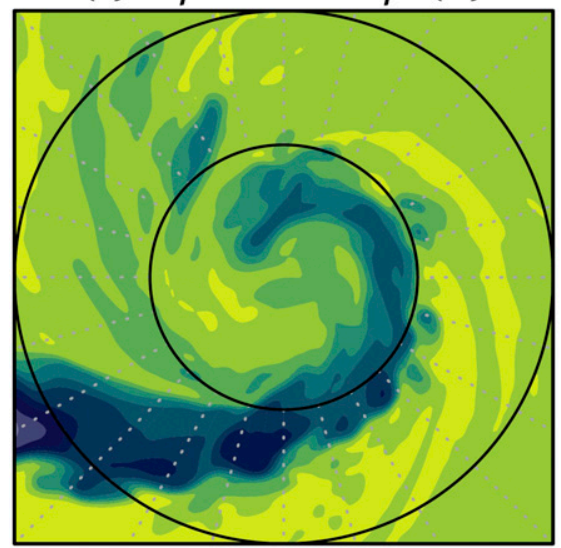

(b) Vorticity $\left(10^{-4} s^{-1}\right)$
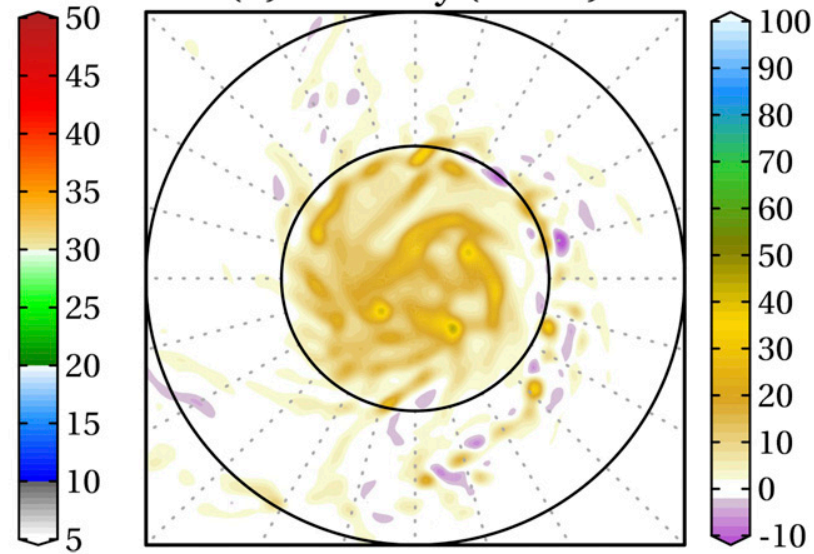

(d) TCWC $\left(\mathrm{kg} \mathrm{m}^{-2}\right)$
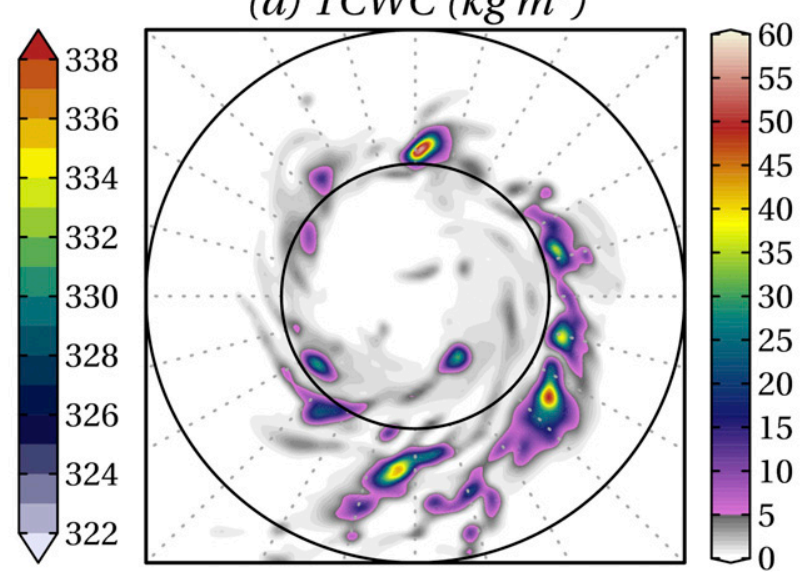

FIG. 12. As in Fig. 11, but for 69 h, 15 min.

coincident radial at the same time, capturing the eyewall, a primary rainband, and a secondary rainband (Fig. 15). Figure 16 compares convective cells in both the sheared TC and the control TC. In the sheared TC, the updraft isolated is the strongest (i.e., largest vertical velocity magnitude) tower from Fig. $14 \mathrm{c}$ at $290^{\circ}$. In terms of radial-vertical streamlines, the tower in the TCA appears to have two separate components. We should note that streamlines do not necessarily indicate parcel trajectories but indicate the combined motion of the updraft on the TC's secondary circulation. At low levels, streamlines converge at $42 \mathrm{~km}$ before moving upward and outward. Some streamlines converge at 5-km height and $60-\mathrm{km}$ radius. Above that, the streamlines indicate a second region of vertical motion. In the control (Fig. 16b), none of the updrafts has this type of vertical structure. Another factor that also separates this tower is the buoyancy structure in the vertical, where thermal buoyancy (e.g., Eastin et al. 2005) is defined as

$$
B=g \frac{\theta_{\rho}^{\prime}}{\overline{\theta_{\rho}}}
$$

To separate the updrafts from the TC, the denominator in (5) is the 2.5-h time-mean density potential temperature. Strictly speaking, the perturbation defined here (i.e., the updraft) is the deviation from the time-mean vortex and the background (see appendix B for an explanation of this term). Additionally, we are only focusing on the forcing at this particular instant, as buoyancy is active throughout a parcel's entire ascent. "Dynamic buoyancy" (Eastin et al. 2005) was also calculated, but its contributions are an order of magnitude smaller. Given the definition of density potential temperature (see appendix A), hydrometeor drag is implicitly factored into (5). In the sheared case (Fig. 16c), the tower, simply characterized here as a localized area within the $2.5 \mathrm{~m} \mathrm{~s}^{-1}$ vertical motion contour, is thermally buoyant from 2- to $14-\mathrm{km}$ height. In the control 
(a) DPT Anomaly $(K)$

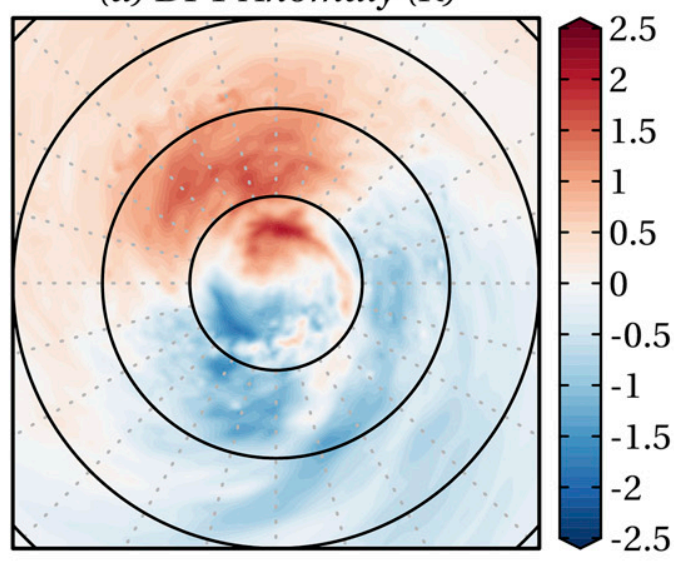

(c) TR DPT Anomaly (K)

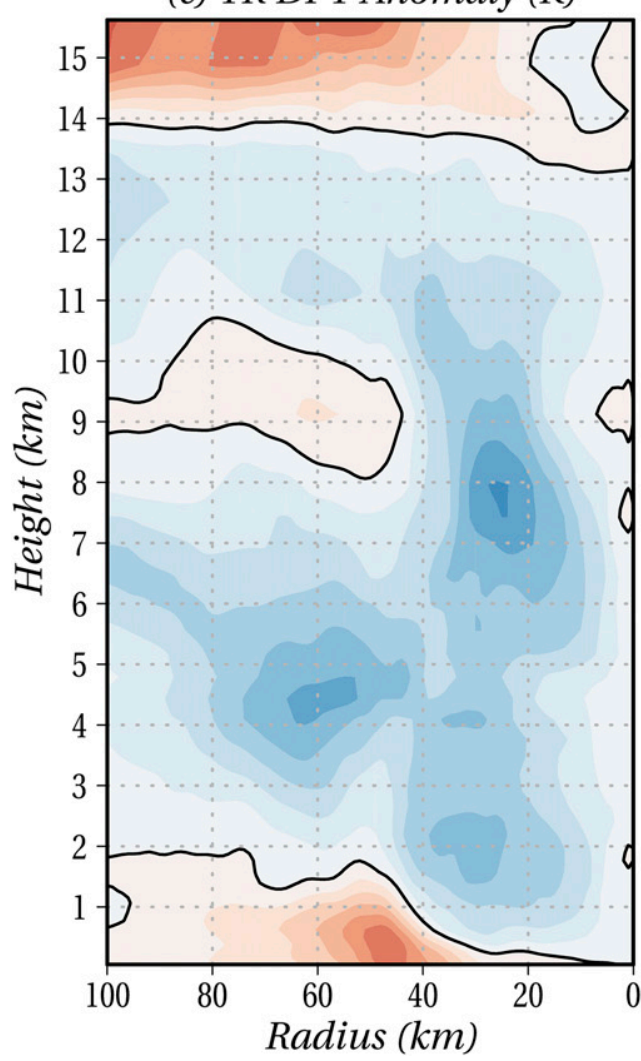

(b) Vertical Mass Flux $\left(\mathrm{kg} \mathrm{m}^{-2} \mathrm{~s}^{-1}\right)$
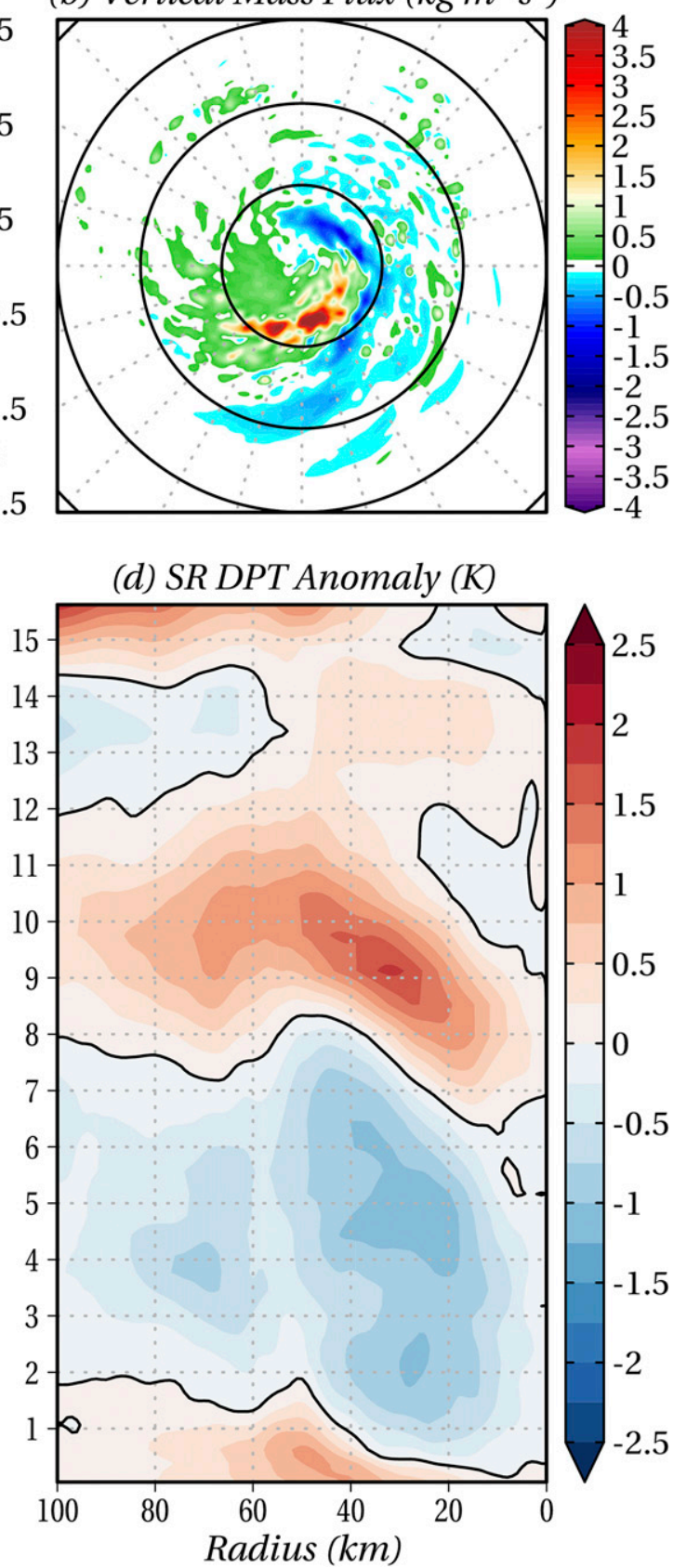

FIG. 13. Tilt-relative 2.5-h-smoothed DPT anomaly as deviation from (a) azimuthal mean, and (b) vertical mass flux centered around the PVV120 center at 6-km height at $65 \mathrm{~h}, 30 \mathrm{~min}$. Black circles are radii at every $50 \mathrm{~km}$. (c) Tilt-relative and (d) storm-relative (surface centered) DPT anomalies averaged over $2.5 \mathrm{~h}$ and $90^{\circ}$ downshear left. Black contour in (c),(d) is $0 \mathrm{~K}$.

simulation (Fig. 16d), the three towers here all display different buoyancy structures from one another, and none of them has the same structure as the tower in the sheared TC. Finally, in terms of vorticity, the tower in the sheared TC has an apparent dual lobe structure. In the tower in the sheared TC (Fig. 16e), the largest vorticity values are confined with the central portions of the updraft up to approximately $6 \mathrm{~km}$, indicative of a "vortical" structure (e.g., Hendricks et al. 2004). Beginning at $5 \mathrm{~km}$ and reaching $13 \mathrm{~km}$ in height, there is a secondary lobe of vorticity on the inner radial edge of the updraft. None of the updrafts in the control (Fig. 16f) 
(a) $065 \mathrm{hr} 00 \mathrm{~min}$

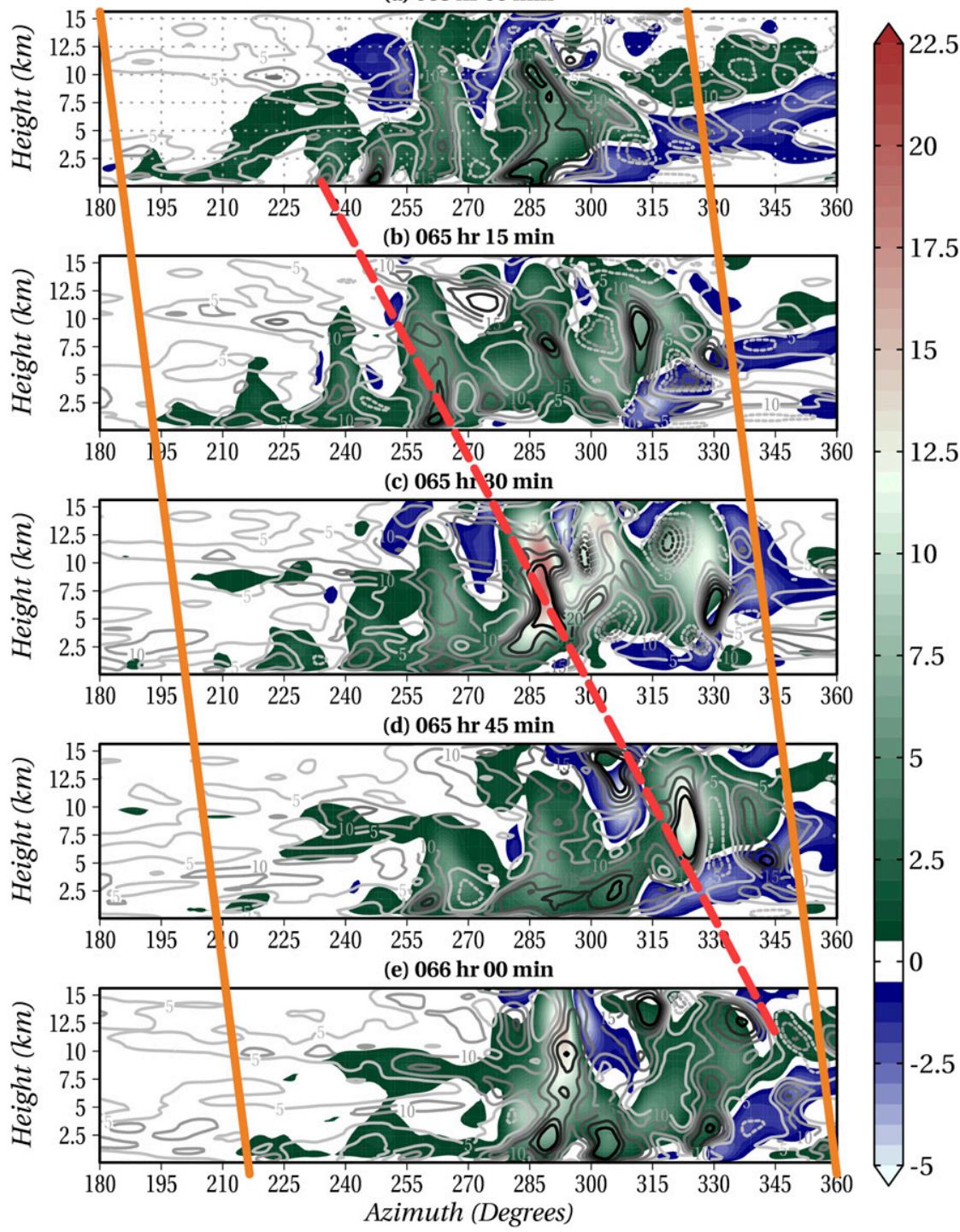

FIG. 14. Storm-relative azimuth-height cross section of vertical velocity ( $\mathrm{m} \mathrm{s}^{-1}$, shaded) and vertical vorticity $\left(10^{-4} \mathrm{~s}^{-1}\right.$; contour) in a TCA averaged between $45-$ and $55-\mathrm{km}$ radius at (a) $65 \mathrm{~h}$; (b) $65 \mathrm{~h}, 15 \mathrm{~min}$; (c) $65 \mathrm{~h}, 30 \mathrm{~min}$; (d) $65 \mathrm{~h}, 45 \mathrm{~min}$; and (e) $66 \mathrm{~h}$. Solid contours are positive vorticity; dashed contours are negative vorticity. Both are at $5 \times 10^{-4} \mathrm{~s}^{-1}$ intervals. Orange lines frame the convection associated with the slow-moving tilt; dashed red line traces the azimuthal motion of a continuous convective tower.

has this vorticity structure. The tower in the sheared simulation most resembles that of the inner rainband, originating at a radius of $32 \mathrm{~km}$ in the control simulation; however, the buoyancy structure and the vorticity structure between the two towers are different.

\section{Discussion and summary}

A tilt-modulated convective asymmetry (TCA) in a sheared, rapidly intensifying tropical cyclone (TC) in an idealized model (CM1) has been investigated in this study. The vertical structure of the background environmental field is found to have a profound effect on the evolution of the TC, and this structure may in part allow for the development of these TCAs (see Fig. 1 from Part I). Precipitation analyses, diagnosed using total condensed water of the column, show a very asymmetric pattern, not unexpected with a sheared TC. The localized maxima, however, appear to be associated with the tilt of the midlevel vortex center. Eventually, this midlevel center migrates all the way back from a downshear maximum at $24 \mathrm{~h}$ to almost completely upshear near $72 \mathrm{~h}$. 


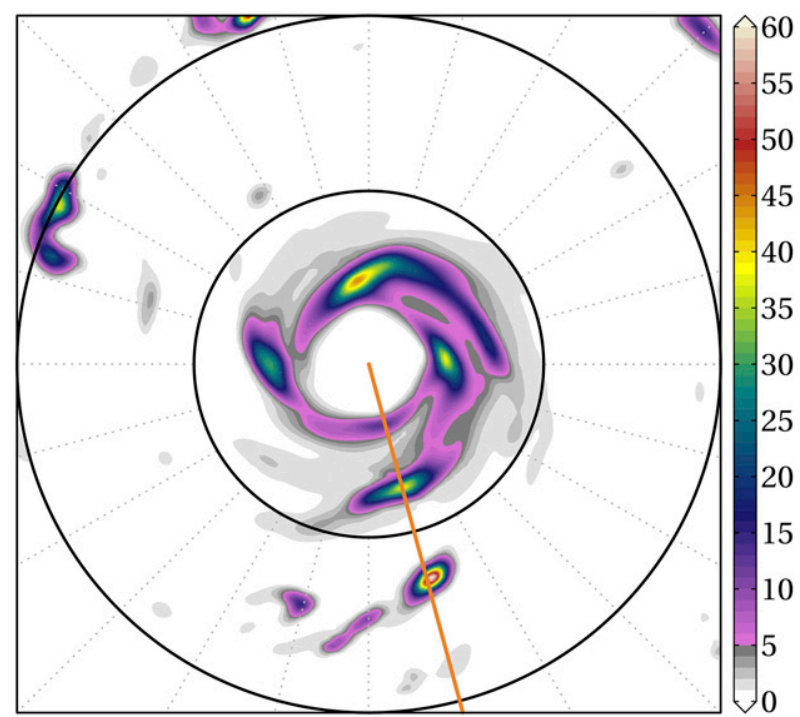

FIG. 15. TCWC $\left(\mathrm{kg} \mathrm{m}^{-2}\right)$ from the control simulation at $87 \mathrm{~h}$, $15 \mathrm{~min}$. Orange line is the azimuth of the radial-height cross section for the comparison in Fig. 16.

The midlevel vortex then orbits a new axis left of shear. Using a similar filter as the one used in Part I, the tilt evolution can be divided into a longer realignment precession (days) and shorter nutations (hours). Tying this to the results of Part I, the upshear migration of the cloud shield (as a proxy for heaviest precipitation) over the span of 1-2 days is related to the longer precession of the vortex. The subsequent $4-8$-h pulsing of the cold cloud-top temperatures in Hernan, Guillermo, Norbert, and the rest are tied to the higher-frequency nutations of the vortex tilt superposed on the larger, longer precession. These features are identified as the TCAs.

TCAs are a collection of vortical hot towers (Hendricks et al. 2004) in that the individual cells initiate in the boundary layer, and the resultant vertical motion appears coupled with vorticity. Upon reaching the midlevels of the TCA, the vertical motion shifts radially and azimuthally. At low levels, in an azimuthal sense, updrafts tilt with the mean flow. At mid- and upper levels, updrafts tilt slightly into the mean flow. At low levels, in a radial sense, the updrafts move outward radially, as would be expected from a typical TC. Once these updrafts reach midlevels, they become more vertical. These updrafts are buoyant throughout their existences, due to their ascents within a vortex-scale cold anomaly induced by the tilt of the vortex. This is a key component that separates the TCA from other convective features.

Drawing on the results from this study along with the satellite observations in Part I, a TCA can be characterized in the following way. A TCA is an azimuthally localized, left-of-shear collection of buoyant updrafts modulated by the nutation of the tilt of the vortex whose temporal period is between 4 and $8 \mathrm{~h}$. These features appear when the nutation and the precession magnitudes are approximately equivalent and when the precession is left of shear. In observational satellite imagery, this is manifested as an upshear-expanding cloud feature encapsulated by the $-70^{\circ} \mathrm{C}$ isotherm originating in the left-of-shear side of the TC that is generally responsible for a $5000-20000-\mathrm{km}^{2}$ change in cloud coverage colder than $-70^{\circ} \mathrm{C}$, whose coldest cloud-top temperatures are below $-80^{\circ} \mathrm{C}$, and that expands radially outward at a speed of approximately $20 \mathrm{~m} \mathrm{~s}^{-1}$. Structurally, this can be summarized by the schematic in Fig. 17, where the midlevel center (gray " $\mathrm{M}$ ") is in the middle of a nutation with a collection of buoyant cells within it. This schematic can be thought of a top-down perspective of the original schematic (Fig. 1 from Part I).

A significant question is where TCAs fit within the larger knowledge base of sheared TCs. Recent sheared TC research has focused on the upshear migration of convection (e.g., Zawislak et al. 2016), while other studies have discussed the movement of the tilt (Finocchio et al. 2016; Zhang and Tao 2013), but this study explicitly relates the movement of the tilt-separating the movement into the longer precession and shorter nutations- to the modulation of convection. Moreover, what separates TCAs from MCSs, discussed by Rappin and Nolan (2012), is in the specific cause and the initialization. Rappin and Nolan's initial condition is a starting midlevel vortex. Here, it is a low-level vortex. They only loosely associated it with the tilt. Here, we demonstrated that the convective periodicity is associated with a vortex-specific asymmetry. Additionally, in terms of the importance of the wind profile, Tao and Zhang (2015) indicated that with their wind profile (essentially linear with height), they could not get development when the shear was $7.5 \mathrm{~m} \mathrm{~s}^{-1}$ with SSTs of $27^{\circ} \mathrm{C}$. Here, development and RI at a vertical wind shear of $7.5 \mathrm{~m} \mathrm{~s}^{-1}$ and SSTs of $27.5^{\circ} \mathrm{C}$ are supported by observations from Part I, and we have corroborated that it is not only the magnitude, but also the vertical structure of the background winds that is important. And finally, in a multitude of papers (e.g., Braun et al. 2006; Hazelton et al. 2017), the focus of convective modulation has primarily been on lowlevel forcing such as mesovortices. This work demonstrates that there exists a spatiotemporal component of the tilt evolution that also modulates convection. While we realize that there are several necessary preconditions for this behavior, we feel TCAs are nonetheless distinct enough to merit identifying them as new phenomena, recalling that they also have distinct satellite features (see Part I). 
(a) $\mathrm{v}_{\mathrm{t}}\left(\mathrm{ms}^{-1}\right), \mathrm{u}_{\mathrm{r}}$ and $\mathrm{w}$ (stream)

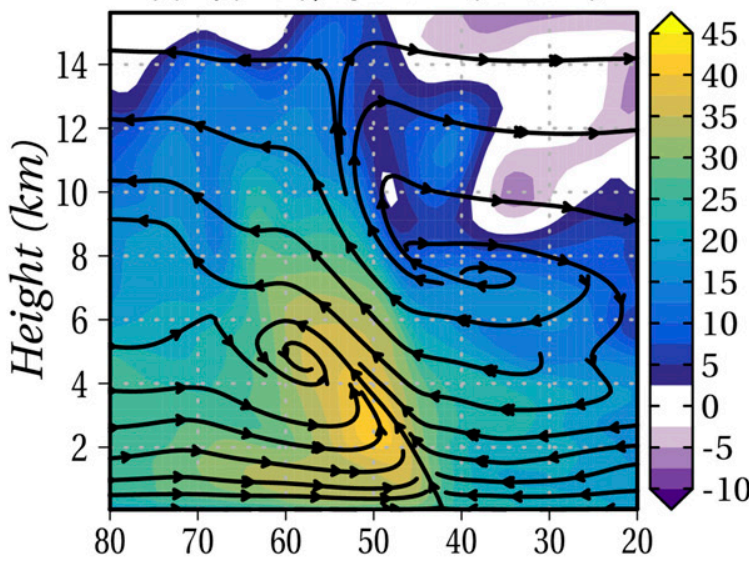

(c) Th. Buoy. $\left(10^{-2} \mathrm{~ms}^{-2}\right), \mathrm{w}\left(\mathrm{ms}^{-1}\right)$

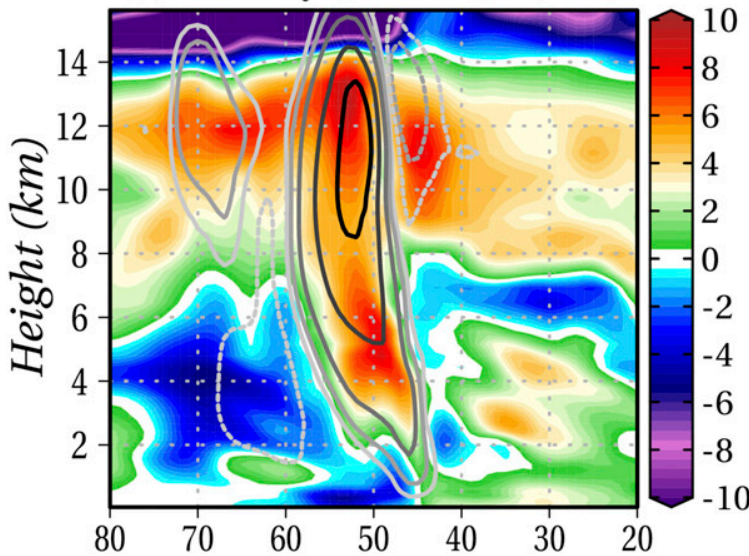

(e) Vorticity $\left(10^{-4} \mathrm{~s}^{-1}\right), \mathrm{w}\left(\mathrm{ms}^{-1}\right)$

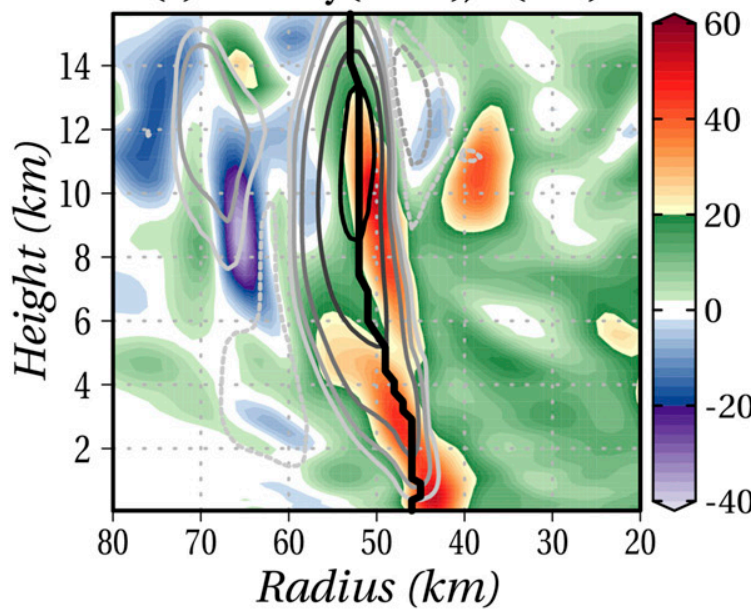

(b) $\mathrm{v}_{\mathrm{t}}\left(\mathrm{ms}^{-1}\right), \mathrm{u}_{\mathrm{r}}$ and $\mathrm{w}$ (stream)

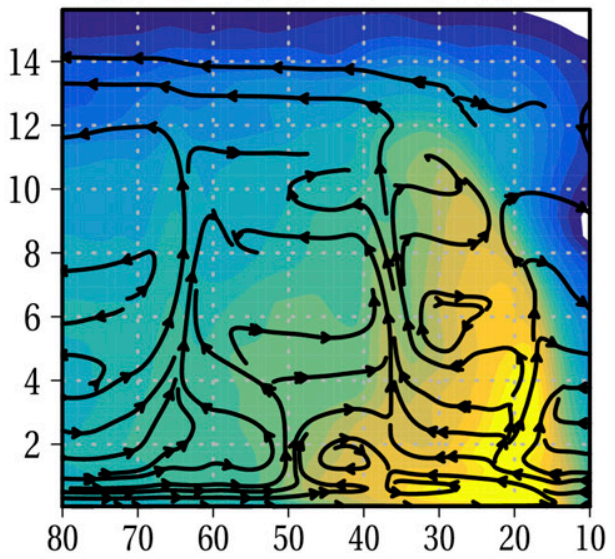

(d) Th. Buoy. $\left(10^{-2} \mathrm{~ms}^{-2}\right), \mathrm{w}\left(\mathrm{ms}^{-1}\right)$

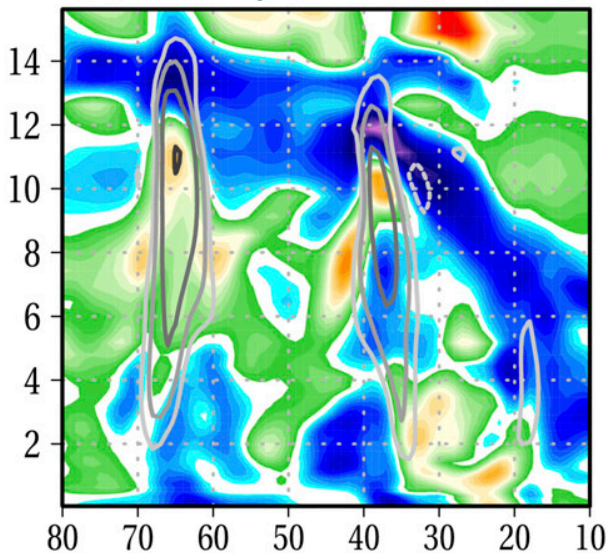

(f) Vorticity $\left(10^{-4} \mathrm{~s}^{-1}\right), \mathrm{w}\left(\mathrm{ms}^{-1}\right)$

FIG. 16. Radial-height cross section of (a),(b) $10^{\circ}$-averaged winds; (c),(d) thermal buoyancy (see appendix B) with vertical velocity overlaid; and (e),(f) total vertical vorticity with vertical velocity overlaid along with the radius of maximum vertical motion for each updraft for (left) the sheared TC's updraft in Fig. 14c located at $287^{\circ}$ and (right) the control TC's collection of updrafts identified in Fig. 15. Vertical velocity contours are 2.5, 5, 10, 20, and $30 \mathrm{~m} \mathrm{~s}^{-1}$, respectively.

From a more theoretical perspective, Schecter (2015) and Reasor and Montgomery (2015) used a series of idealized and linearized simulations to demonstrate that reduced static stability in the core of the TC, serving as a proxy for convection, acts to reduce the tilt of the vortex. Schecter (2015) then separated the primary tilt mode from an inner wobble mode. We believe that the precession-nutation separation described herein is 


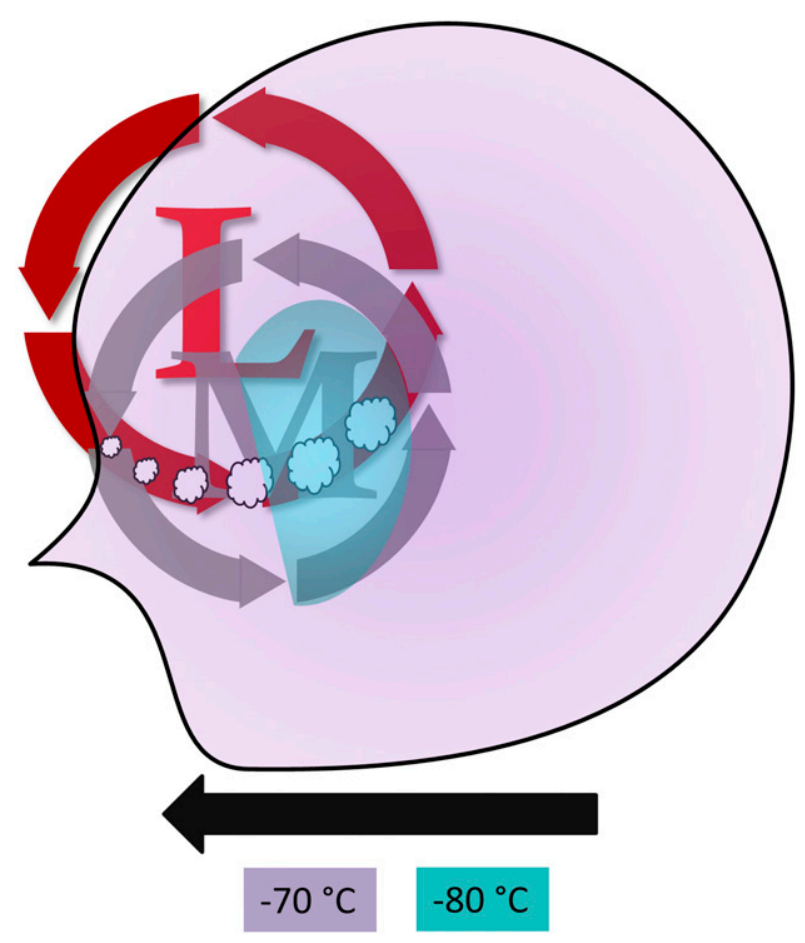

FIG. 17. Schematic illustrating the orientation of the lowlevel center (red "L" and arrows) and the midlevel center (gray " $\mathrm{M}$ " and arrows) underneath a TCA (transparent lavenders and teals, corresponding to brightness temperatures) in the midst of a tilt nutation after the precession has reached left of shear. Note the upshear-left orientation of the midlevel center. Also included are indications of growing convective towers underneath the midlevel tilt (white clouds). Large black arrow is shear direction.

most likely the same. Schecter (2015) demonstrated that the nutations are not affected by reduced static stability; therefore, it might be related to an intrinsic dynamic property of the vortex. Given the periodicities found in observations and in this simulation, the nutation is likely associated with the size and the structure of the vortex. Our idealized vortex here is large $(600 \mathrm{~km})$, larger than the TCs in observations based on cloud shield size (Part I). That could be part of the reason why the nutation is $9 \mathrm{~h}$. The periodicities of the two largest TCs from Part I, Guillermo and Joaquin, were $8 \mathrm{~h}$.

There are several implications for the convective activity at all levels in the storm, facets of this evolution that we will endeavor to study in following manuscripts. Preliminarily, these TCAs appear to serve two primary purposes. First, at upper levels, the enhanced outflow exits the TC upshear, thus diverting the upper-level environmental winds and locally reducing the shear forcing while also creating an outflow channel. At midlevels, a midlevel center is strengthened by the accumulation of tilting vorticity, much in the same way that the rotating convective paradigm asserts that accumulated stretching vorticity by individual cells strengthens the lowlevel vorticity. This then induces an increase in mass flux under the tilt. Future research will explore the spinup and the rapid intensification that occurs in this class of TCs.

Acknowledgments. A large portion of this research was performed as a National Research Council postdoctoral scientist at the Naval Research Laboratory Marine Meteorology Division in Monterey, California. This research was supported by the Office of Naval Research PE 0601153N as part of the Tropical Cyclone Intensity Departmental Research Initiative (TCI-DRI). DRR would like to acknowledge George Bryan for making the CM1 and the Fortran CAPE subroutine freely available. He would also like to acknowledge Kevin Viner for his time and helpful discussions. We also thank John Knaff, Chris Velden, and one anonymous reviewer for their very helpful comments that greatly improved this work's clarity and content.

\section{APPENDIX A}

\section{Model Initialization Specifics}

The pressure terms in the CM1 equations of motion are cast in Exner form $\Pi$, with the thermodynamic variable in the equations of motion as density potential temperature $\theta_{\rho}$. Each is defined:

$$
\begin{aligned}
\Pi & \equiv\left(\frac{p}{p_{\text {ref }}}\right)^{R_{d} / c_{p}}, \\
\theta_{\rho} & \equiv \theta\left(\frac{1+r_{v} / \varepsilon}{1+r_{v}+r_{c}+r_{r}+r_{s}+r_{i}+r_{g}}\right),
\end{aligned}
$$

where $p_{\text {ref }}$ is $1000 \mathrm{hPa}, R_{d}$ is the dry gas constant, $c_{p}$ is the heat capacity of dry air, $\varepsilon$ is the ratio of the gas constants, and each $r$ is a mixing ratio of a different species of water (vapor, cloud, rain, snow, ice, and graupel). The derivation found in appendix B of Ryglicki (2015) for the linearized thermal wind balance equations can be generalized to yield the two balance perturbation equations for any initial wind field that varies only in height:

$$
\Pi_{\mathrm{TWB}}=\frac{\left(f_{0}+\beta y\right) v x-\left[f_{0}+(\beta / 2) y\right] u y}{c_{p} \theta_{\rho 0}},
$$




$$
\theta_{\rho, \mathrm{TWB}}=\frac{\theta_{\rho 0}}{g}\left(\begin{array}{l}
\left\{\left(f_{0}+\beta y\right) x \frac{\partial v}{\partial z}-\left[f_{0}+(\beta / 2) y\right] y \frac{\partial u}{\partial z}\right\} \\
+\frac{N_{0}^{2}}{g}\left\{\left[f_{0}+(\beta / 2) y\right] u y-\left(f_{0}+\beta y\right) v x\right\}-\left[\left(e_{0}+\beta_{e} y\right) u\right]
\end{array}\right),
$$

where the subscript TWB indicates that these are perturbations associated with the background in thermal wind balance. In (A2) and (A3), $\theta_{\rho 0}$ is the base state density potential temperature; $f_{0}$ and $e_{0}$ are the fixedlatitude Coriolis parameters; $\beta$ and $\beta_{e}$ are the meridional variations for their respective Coriolis parameters; $u$ and $v$ are the prescribed components of the background flow; $x$ and $y$ are domain values, generally taken to be distance from the origin at the center of the domain; and $N_{0}^{2}$ is the squared Brunt-Väisälä frequency of the base state. Parameter $g$ corresponds to gravity. All "base state" variables vary with height only. Equations (A2) and (A3) are formulated as an anelastic approximation to thermal wind balance. For the simulations here, only the zonal wind is prescribed, thereby allowing the zonal boundaries to be periodic and the meridional boundaries to be rigid walls with Rayleigh damping layers of $150-\mathrm{km}$ width to form a channel model. While we are aware of the ability to use the CM1's version of point downscaling (Nolan 2011), we have chosen to include the more complete background effects. The caveat with using Eqs. (A2) and (A3) is that the resulting horizontal gradients must not result in saturation, as this would complicate the computation of $N^{2}$. This is only a concern at lowest levels, where base-state relative humidity is high. Since the environmental winds are constant in the lowest $1 \mathrm{~km}$, the perturbations are small; thus, the environment remains unsaturated. In addition, using a scale analysis, it can be shown that the second bracket inside the larger braces of (A3) - the term that is multiplied by the static stabilityis an order of magnitude smaller than the first bracket.

The features and settings of the CM1 are summarized in Table A1. The horizontal breadth of the domain is $5998 \mathrm{~km} \times 4095 \mathrm{~km}$. The grid spacing of the innermost $1000 \mathrm{~km} \times 300 \mathrm{~km}$ is $2 \mathrm{~km}$. The grid is slowly stretched to $15 \mathrm{~km}$ at the zonal boundaries and to $12 \mathrm{~km}$ at the meridional boundaries, resulting in 1088 grid points in the zonal direction and 656 grid points in the meridional direction. Vertical grid spacing is $100 \mathrm{~m}$ at the lowest model level and stretched to $750 \mathrm{~m}$ at $16-\mathrm{km}$ height. The tropopause $\left(N^{2}=2 \times 10^{-4} \mathrm{~s}^{-1}\right)$ is approximately $14 \mathrm{~km}$. A Rayleigh damping layer is present from $20-\mathrm{km}$ height to the model top at $26.5 \mathrm{~km}$, resulting in 56 grid points in the vertical. The model is set to an $f$ plane at approximately $20^{\circ} \mathrm{N}$. The model TCs are initialized using the standard Rotunno and Emanuel (1987) method, with a radial extent of $600 \mathrm{~km}$, an initial RMW of $100 \mathrm{~km}$, an initial surface maximum mean tangential wind of $20 \mathrm{~m} \mathrm{~s}^{-1}$, and an initial maximum height of $10 \mathrm{~km}$. For the simulations in the presence of vertical wind shear, the TCs are initialized slightly outside the highest-resolution grid at $550 \mathrm{~km}$ east of the origin to account for the advection and tilt into the highest-resolution area. For the nonsheared control, the storm is initialized at the origin. Output is produced every $15 \mathrm{~min}$ out to a final model time of $144 \mathrm{~h}$ ( 6 days). The YSU boundary layer parameterization scheme (Hong et al. 2006) is used. In lieu of a radiation scheme, Newtonian cooling, capped at $2 \mathrm{Kday}^{-1}$, is enabled. This "permanent night" setting eliminates the diurnal forcing (Dunion et al. 2014; Navarro and Hakim 2016) and simplifies the analysis. All other model settings, including the Morrison double-moment microphysics scheme (Morrison et al. 2009) and the new pressure-dependent parameterized horizontal turbulent length scale, are default values. Sensitivity tests varying these parameters, including storm structure characteristics and microphysical options, were not performed and are reserved for a future study.

For the sheared cases with the Gaussian wind profile,

$$
u(z)=u_{0} \exp \left[\frac{-\left(z-z_{p}\right)^{2}}{2 z_{w}^{2}}\right]+u_{b}
$$

$u_{0}$ is the amplitude (equal to -7.5 or $-11.5 \mathrm{~m} \mathrm{~s}^{-1}$ ), $z$ is the model level height, $z_{p}$ is the level of peak winds $(13 \mathrm{~km}), z_{w}$ is the decay width in the vertical $(2.5 \mathrm{~km})$, and $u_{b}$ is a base background flow $\left(-1 \mathrm{~m} \mathrm{~s}^{-1}\right)$. For the cosine wind profile,

$$
\begin{aligned}
& u(z)=u_{0}+u_{b}, \quad z \leq z_{\mathrm{st}} \\
& u(z)=u_{0} \cos \left[\pi \frac{\left(z-z_{\mathrm{st}}\right)}{\left(z_{\mathrm{top}}-z_{\mathrm{st}}\right)}\right]+u_{b}, \quad z_{\mathrm{st}}<z<z_{\mathrm{top}} \\
& u(z)=-u_{0}+u_{b}, \quad z_{\mathrm{top}} \leq z
\end{aligned}
$$

$u_{0}$ is again the amplitude (equal to $3.75 \mathrm{~m} \mathrm{~s}^{-1}$ ), $z$ is the model level height, $z_{\mathrm{st}}$ is the model height that begins the cosine blend zone $(1.5 \mathrm{~km}), z_{\text {top }}$ is the top of the 
TABLE A1. CM1 initialization settings.

\begin{tabular}{ll}
\hline \hline Domain, horizontal & $5998 \mathrm{~km} \times 4095 \mathrm{~km}$ \\
& $1088 \times 656$ grid points \\
& $2 \mathrm{~km} d x, d y$ in innermost $1000 \mathrm{~km} \times$ \\
& $300 \mathrm{~km}$ \\
& $d x$ stretched to $15 \mathrm{~km} ; d y$ to $12 \mathrm{~km}$ \\
& Rigid walls N/S; periodic E/W \\
& $150-\mathrm{km}$ Rayleigh damping on walls \\
& $0-26.5 \mathrm{~km}$ \\
& 56 grid points \\
& $100 \mathrm{~m} d z$ stretched to $750 \mathrm{~m}$ at $16 \mathrm{~km}$ \\
Domain, vertical & Rayleigh damping from $20 \mathrm{~km}$ to top \\
& Moist tropical (Dunion 2011$)$ \\
& Rotunno and Emanuel $(1987)$ method \\
& $600-\mathrm{km}$ extent \\
Sounding & RMW $=150 \mathrm{~km}$ \\
TC initialization & MMTW $=20 \mathrm{~m} \mathrm{~s}{ }^{-1}$ \\
& Height $=10 \mathrm{~km}$ \\
& 15 min, up to $144 \mathrm{~h}$ \\
Output frequency & Newtonian cooling $\left(2 \mathrm{~K} \mathrm{day}^{-1}\right)$ \\
Physics & Morrison et al. (2009) microphysics \\
& YSU boundary layer \\
& $27.5^{\circ} \mathrm{C}$ SSTs, constant \\
& Parameterized horizontal turbulence \\
\hline
\end{tabular}

blend zone $(13 \mathrm{~km})$, and $u_{b}$ is the base background flow $\left(-4.75 \mathrm{~m} \mathrm{~s}^{-1}\right)$.

\section{APPENDIX B}

\section{Updraft Thermal Buoyancy in the CM1}

Equation (5) is the calculation shown in Figs. 16d and $16 \mathrm{e}$ to express the buoyancy of a parcel relative to the vortex plus the background. Technically, this is not how buoyancy is calculated directly in the CM1. In the CM1, the vertical momentum equation, neglecting subgridscale processes and Coriolis, is

$$
\frac{D w}{D t}=-c_{p} \theta \frac{\partial \Pi^{\prime}}{\partial z}+g \frac{\theta_{\rho}^{\prime}}{\theta_{\rho 0}},
$$

where the 0 denotes the background, the prime indicates deviations from the background state, and $D / D t$ indicates the full Lagrangian time derivative. Note that we are only interested in vertical accelerations determined by temperature and pressure changes, so we are ignoring advection. This also means we are not interested in a full vertical momentum budget (to be explored in a future manuscript).

The second term on the right of (B1) is the "thermal buoyancy" term. Where (5) and (B1) differ is in choice of reference state. In the CM1, there is no explicit relationship between temperature anomalies associated with individual convective cells and the larger temperature anomaly associated with the vortex. To explain this effect numerically, we decompose the righthand side of (B1). We separate the total perturbation (prime) field into the time-mean vortex (tilde) and the perturbations thereof (double prime):

$$
\begin{aligned}
\frac{D w}{D t}= & -c_{p}\left(\theta_{\rho 0}+\tilde{\theta}_{\rho}\right) \frac{\partial \tilde{\Pi}}{\partial z}-c_{p}\left(\theta_{\rho 0}+\tilde{\theta}_{\rho}\right) \frac{\partial \Pi^{\prime \prime}}{\partial z}-c_{p} \theta_{\rho}^{\prime \prime} \frac{\partial \tilde{\Pi}}{\partial z} \\
& -c_{p} \theta_{\rho}^{\prime \prime} \frac{\partial \Pi^{\prime \prime}}{\partial z}+g \frac{\tilde{\theta}_{\rho}}{\theta_{\rho 0}}+g \frac{\theta_{\rho}^{\prime \prime}}{\theta_{\rho 0}} .
\end{aligned}
$$

The first focus will be on the relationship of the two terms that involve no perturbations: the first and fifth terms on the right-hand side. If we assume that the vortex is in hydrostatic balance (Willoughby 1990), then these two terms should balance. This is an effect that can be described by asymmetric balance theory (Shapiro and Montgomery 1993; Reasor et al. 2004). Explicitly, vortex hydrostatic balance is defined:

$$
c_{p}\left(\theta_{\rho 0}+\tilde{\theta}_{\rho}\right) \frac{\partial \tilde{\Pi}}{\partial z}=g \frac{\tilde{\theta}_{\rho}}{\theta_{\rho 0}} .
$$

We now focus on the accelerations on individual convective updrafts, which we assume are nonhydrostatic. From a computational perspective, the vertical acceleration of a convective tower rising in a hydrostatically balanced TC is

$$
\frac{D w}{D t}=-c_{p} \theta_{\rho}^{\prime \prime} \frac{\partial \tilde{\Pi}}{\partial z}+g \frac{\theta_{\rho}^{\prime \prime}}{\theta_{\rho 0}}-c_{p} \theta_{\rho} \frac{\partial \Pi^{\prime \prime}}{\partial z} .
$$

There is an inherent downward force caused by the vertical Exner (pressure) gradient of the vortex assuming a positive updraft thermal anomaly. Using the definition of vortex hydrostatic balance (B3), we can rewrite (B4):

$$
\frac{D w}{D t}=-\frac{g \theta_{\rho}^{\prime \prime} \tilde{\theta}_{\rho}}{\theta_{\rho 0}\left(\theta_{\rho 0}+\tilde{\theta}_{\rho}\right)}+g \frac{\theta_{\rho}^{\prime \prime}}{\theta_{\rho 0}}-c_{p} \theta_{\rho} \frac{\partial \Pi^{\prime \prime}}{\partial z},
$$

rearranging

$$
\frac{D w}{D t}=g \frac{\theta_{\rho}^{\prime \prime}}{\theta_{\rho 0}}\left(1-\frac{\tilde{\theta}_{\rho}}{\theta_{\rho 0}+\tilde{\theta}_{\rho}}\right)-c_{p} \theta_{\rho} \frac{\partial \Pi^{\prime \prime}}{\partial z} .
$$

When creating a common denominator inside the parentheses, a series of cancellations yields the vertical momentum equation:

$$
\frac{D w}{D t}=g \frac{\theta_{\rho}^{\prime \prime}}{\theta_{\rho 0}+\tilde{\theta}_{\rho}}-c_{p} \theta_{\rho} \frac{\partial \Pi^{\prime \prime}}{\partial z} .
$$


The buoyancy expression (5) plotted in Fig. 16 is the first term on the right-hand side of (B7). Explicitly, we are plotting the buoyancy of a parcel relative to a vortex and environment both in their respective hydrostatic balances. This effect is implicitly calculated in the CM1. Physically, on the downtilt side, the vortex temperature anomaly will be lower than on the uptilt side or, hypothetically, in a TC of similar intensity (RMW, etc.) that is not tilted. This reduces the denominator in the first term in (B7), which will then permit the thermal perturbation of the updraft to accelerate the updraft more easily than it would have otherwise. While we choose not to calculate this explicitly, since it is beyond the scope of the paper, if the vortex is not in hydrostatic balance (whether locally or in an azimuthally averaged sense), then the updraft's motion will also be affected by ambient lift/descent associated with departures from hydrostatic balance.

\section{REFERENCES}

Barnes, G. M., and G. J. Stossmeister, 1986: The structure and decay of a rainband in Hurricane Irene (1981). Mon. Wea. Rev., 114, 2590-2601, https://doi.org/10.1175/1520-0493(1986) $114<2590$ :TSADOA $>2.0 . \mathrm{CO} ; 2$.

__ E. J. Zipser, D. Jorgensen, and F. Marks, 1983: Mesoscale and convective structure of a hurricane rainband. J. Atmos. Sci., 40, 2125-2137, https://doi.org/10.1175/1520-0469(1983) $040<2125$ :MACSOA $>2.0 . \mathrm{CO} ; 2$.

—_, J. F. Gamache, M. A. LeMone, and G. J. Stossmeister, 1991: A convective cell in a hurricane rainband. Mon. Wea. Rev., 119, 776-794, https://doi.org/10.1175/1520-0493(1991) $119<0776$ :ACCIAH $>2.0$. CO;2.

Bender, M. A., 1997: The effect of relative flow on the asymmetric structure in the interior of hurricanes. J. Atmos. Sci., 54, 703-724, https://doi.org/10.1175/1520-0469(1997) 054<0703:TEORFO $>2.0 . \mathrm{CO} ; 2$.

Bikos, D., and Coauthors, 2012: Synthetic satellite imagery for realtime high-resolution model evaluation. Wea. Forecasting, 27, 784-795, https://doi.org/10.1175/WAF-D-11-00130.1.

Braun, S. A., M. T. Montgomery, and Z. Pu, 2006: High-resolution simulation of Hurricane Bonnie (1998). Part I: The organization of eyewall vertical motion. J. Atmos. Sci., 63, 19-42, https://doi.org/10.1175/JAS3598.1.

Bryan, G. H., and J. M. Fritsch, 2002: A benchmark simulation for moist nonhydrostatic numerical models. Mon. Wea. Rev., 130, 2917-2928, https://doi.org/10.1175/1520-0493(2002)130<2917: ABSFMN $>2.0 . \mathrm{CO} ; 2$.

Carrió, G. G., H. Jiang, and W. R. Cotton, 2005: Impact of aerosol intrusions on Arctic boundary layer clouds. Part I: 4 May 1998 case. J. Atmos. Sci., 62, 3082-3093, https://doi.org/10.1175/ JAS3454.1.

Corbosiero, K. L., and J. Molinari, 2003: The relationship between storm motion, vertical wind shear, and convective asymmetries in tropical cyclones. J. Atmos. Sci., 60, 366-376, https://doi.org/ 10.1175/1520-0469(2003)060<0366:TRBSMV>2.0.CO;2.

Cram, T. A., J. Persing, M. T. Montgomery, and S. A. Braun, 2007: A Lagrangian trajectory view on transport and mixing processes between the eye, eyewall, and environment using a high-resolution simulation of Hurricane Bonnie
(1998). J. Atmos. Sci., 64, 1835-1856, https://doi.org/10.1175/ JAS3921.1.

Dee, D. P., and Coauthors, 2011: The ERA-Interim reanalysis: Configuration and performance of the data assimilation system. Quart. J. Roy. Meteor. Soc., 137, 553-597, https://doi.org/ 10.1002/qj.828.

DeHart, J. C., R. A. Houze, and R. F. Rogers, 2014: Quadrant distribution of tropical cyclone inner-core kinematics in relation to environmental shear. J. Atmos. Sci., 71, 2713-2732, https://doi.org/10.1175/JAS-D-13-0298.1.

DeMaria, M., 1996: The effect of vertical shear on tropical cyclone intensity change. J. Atmos. Sci., 53, 2076-2088, https://doi.org/ 10.1175/1520-0469(1996)053<2076:TEOVSO > 2.0.CO;2.

- M. Mainelli, L. K. Shay, J. A. Knaff, and J. Kaplan, 2005: Further improvements to the Statistical Hurricane Intensity Prediction Scheme (SHIPS). Wea. Forecasting, 20, 531-543, https://doi.org/10.1175/WAF862.1.

Dunion, J. P., 2011: Rewriting the climatology of the tropical North Atlantic and Caribbean Sea atmosphere. J. Climate, 24, 893908, https://doi.org/10.1175/2010JCLI3496.1.

— C. D. Thorncroft, and C. S. Velden, 2014: The tropical cyclone diurnal cycle of mature hurricanes. Mon. Wea. Rev., 142, 3900-3919, https://doi.org/10.1175/MWR-D-13-00191.1.

Eastin, M. D., W. M. Gray, and P. G. Black, 2005: Buoyancy of convective vertical motions in the inner core of intense hurricanes. Part II: Case studies. Mon. Wea. Rev., 133, 209-227, https://doi.org/10.1175/MWR-2849.1.

Elsberry, R. L., and R. A. Jeffries, 1996: Vertical wind shear influences on tropical cyclone formation and intensification during TCM-92 and TCM-93. Mon. Wea. Rev., 124, 1374-1387, https:// doi.org/10.1175/1520-0493(1996)124<1374:VWSIOT>2.0.CO;2.

Finocchio, P. M., S. J. Majumdar, D. S. Nolan, and M. Iskandarani, 2016: Idealized tropical cyclone responses to the height and depth of environmental vertical wind shear. Mon. Wea. Rev., 144, 2155-2175, https://doi.org/10.1175/MWR-D-15-0320.1.

Frank, W. M., and E. A. Ritchie, 1999: Effects of environmental flow upon tropical cyclone structure. Mon. Wea. Rev., 127, 2044-2061, https://doi.org/10.1175/1520-0493(1999)127<2044: EOEFUT $>2.0 . \mathrm{CO} ; 2$.

_, and _ 2001: Effects of vertical wind shear on the intensity and structure of numerically simulated hurricanes. Mon. Wea. Rev., 129, 2249-2269, https://doi.org/10.1175/1520-0493(2001) $129<2249$ :EOVWSO $>2.0$. CO 2 .

Ge, X., T. Li, and M. Peng, 2013: Effects of vertical shears and midlevel dry air on tropical cyclone developments. J. Atmos. Sci., 70, 3859-3875, https://doi.org/10.1175/JAS-D-13-066.1.

Grasso, L. D., M. Sengupta, J. F. Dostalek, R. Brummer, and M. DeMaria, 2008: Synthetic satellite imagery for current and future environmental satellites. Int. J. Remote Sens., 29, 43734384, https://doi.org/10.1080/01431160801891820.

Gray, W. M., 1968: Global view of the origin of tropical disturbances and storms. Mon. Wea. Rev., 96, 669-700, https://doi.org/10.1175/ 1520-0493(1968)096<0669:GVOTOO > 2.0.CO;2.

Hazelton, A. T., R. F. Rogers, and R. E. Hart, 2017: Analyzing simulated convective bursts in two Atlantic hurricanes. Part I: Burst formation and development. Mon. Wea. Rev., 145, 30733094, https://doi.org/10.1175/MWR-D-16-0267.1.

Hendricks, E. A., M. T. Montgomery, and C. A. Davis, 2004: The role of "vortical" hot towers in the formation of Tropical Cyclone Diana (1984). J. Atmos. Sci., 61, 1209-1232, https://doi.org/10.1175/ 1520-0469(2004)061<1209:TROVHT>2.0.CO;2. 
Hodyss, D., and D. S. Nolan, 2008: The Rossby-inertia-buoyancy instability in baroclinic vortices. Phys. Fluids, 20, 096602, https:// doi.org/10.1063/1.2980354.

Hong, S. Y., Y. Noh, and J. Dudhia, 2006: A new vertical diffusion package with an explicit treatment of entrainment processes Mon. Wea. Rev., 134, 2318-2341, https://doi.org/10.1175/ MWR3199.1.

Hoskins, B. J., M. E. McIntyre, and A. W. Robertson, 1985: On the use and significance of isentropic potential vorticity maps. Quart. J. Roy. Meteor. Soc., 111, 877-946, https://doi.org/ 10.1002/qj.49711147002.

Houze, R. A., 2010: Clouds in tropical cyclones. Mon. Wea. Rev., 138, 293-344, https://doi.org/10.1175/2009MWR2989.1.

Jin, Y., and Coauthors, 2014: The impact of ice phase cloud parameterizations on tropical cyclone prediction. Mon. Wea. Rev., 142, 606-625, https://doi.org/10.1175/MWR-D-13-00058.1.

Jones, S. C., 1995: The evolution of vortices in vertical shear. I: Initially barotropic vortices. Quart. J. Roy. Meteor. Soc., 121, 821-851, https://doi.org/10.1002/qj.49712152406.

- 2000: The evolution of vortices in vertical shear. III: Baroclinic vortices. Quart. J. Roy. Meteor. Soc., 126, 3161-3185, https://doi.org/10.1002/qj.49712657009.

Kaplan, J., M. DeMaria, and J. A. Knaff, 2010: A revised tropical cyclone rapid intensification index for the Atlantic and eastern North Pacific basins. Wea. Forecasting, 25, 220-241, https://doi.org/10.1175/2009WAF2222280.1.

Knaff, J. A., S. A. Seseske, M. DeMaria, and J. L. Demuth, 2004: On the influences of vertical wind shear on symmetric tropical cyclone structure derived from AMSU. Mon. Wea. Rev., 132, 2503-2510, https://doi.org/10.1175/1520-0493(2004)132<2503: OTIOVW $>2.0 . \mathrm{CO} ; 2$.

Liang, X., and J. C. L. Chan, 2005: The effects of the full Coriolis force on the structure and motion of a tropical cyclone. Part I: Effects due to vertical motion. J. Atmos. Sci., 62, 3825-3830, https://doi.org/10.1175/JAS3545.1.

Marks, F. D., R. A. Houze, and J. F. Gamache, 1992: Dual-aircraft investigation of the inner core of Hurricane Norbert. Part I: Kinematic structure. J. Atmos. Sci., 49, 919-942, https://doi.org/ 10.1175/1520-0469(1992)049<0919:DAIOTI >2.0.CO;2.

McBride, J. L., and R. Zehr, 1981: Observational analysis of tropical cyclone formation. Part II: Comparison of nondeveloping versus developing systems. J. Atmos. Sci., 38, 1132-1151, https://doi.org/10.1175/1520-0469(1981)038<1132: OAOTCF $>2.0 . \mathrm{CO} ; 2$.

Merrill, R. T., and C. S. Velden, 1996: A three-dimensional analysis of the outflow layer of Supertyphoon Flo (1990). Mon. Wea. Rev., 124, 47-63, https://doi.org/10.1175/1520-0493(1996) $124<0047$ :ATDAOT $>2.0 . \mathrm{CO} ; 2$.

Montgomery, M. T., V. S. Nguyen, J. Persing, and R. K. Smith, 2009: Do tropical cyclones intensify by WISHE? Quart. J. Roy. Meteor. Soc., 135, 1697-1714, https://doi.org/10.1002/qj.459.

Moon, Y., and D. S. Nolan, 2015: Spiral rainbands in a numerical simulation of Hurricane Bill (2009). Part I: Structures and comparisons to observations. J. Atmos. Sci., 72, 164-190, https:// doi.org/10.1175/JAS-D-14-0058.1.

Morrison, H., G. Thompson, and V. Tatarskii, 2009: Impact of cloud microphysics on the development of trailing stratiform precipitation in a simulated squall line: Comparison of one- and two-moment schemes. Mon. Wea. Rev., 137, 991-1007, https:// doi.org/10.1175/2008MWR2556.1.

Navarro, E., and G. Hakim, 2016: Idealized numerical modeling of the diurnal cycle of tropical cyclones. J. Atmos. Sci., 73, 41894201, https://doi.org/10.1175/JAS-D-15-0349.1.
Nguyen, L. T., J. Molinari, and D. Thomas, 2014: Evaluation of tropical cyclone center identification methods in numerical models. Mon. Wea. Rev., 142, 4326-4339, https://doi.org/10.1175/ MWR-D-14-00044.1.

Nolan, D. S., 2011: Evaluating environmental favorableness for tropical cyclone development with the method of point-downscaling. J. Adv. Model. Earth Syst., 3, M08001, https://doi.org/ 10.1029/2011MS000063.

Onderlinde, M. J., and D. Nolan, 2016: Tropical cyclone-relative environmental helicity and the pathways to intensification in shear. J. Atmos. Sci., 73, 869-890, https://doi.org/10.1175/ JAS-D-15-0261.1.

Pattnaik, S., C. Inglish, and T. N. Krishnamurti, 2011: Influence of rain-rate initialization, cloud microphysics, and cloud torques on hurricane intensity. Mon. Wea. Rev., 139, 627-649, https:// doi.org/10.1175/2010MWR3382.1.

Rappin, E. D., and D. S. Nolan, 2012: The effect of vertical shear orientation on tropical cyclogenesis. Quart. J. Roy. Meteor. Soc., 138, 1035-1054, https://doi.org/10.1002/qj.977.

Reasor, P. D., and M. T. Montgomery, 2015: Evaluation of a heuristic model for tropical cyclone resilience. J. Atmos. Sci., 72, 1765-1782, https://doi.org/10.1175/JAS-D-14-0318.1.

,,-- F. D. Marks, and J. F. Gamache, 2000: Low-wavenumber structure and evolution of the hurricane inner core observed by airborne dual-Doppler radar. Mon. Wea. Rev., 128, 1653-1680, https://doi.org/10.1175/1520-0493(2000) $128<1653:$ LWSAEO $>2.0 . \mathrm{CO} ; 2$.

- — , and L. D. Grasso, 2004: A new look at the problem of tropical cyclones in vertical shear flow: Vortex resiliency. J. Atmos. Sci., 61, 3-22, https://doi.org/10.1175/1520-0469(2004) 061<0003:ANLATP $>2.0$. CO;2.

, M. D. Eastin, and J. F. Gamache, 2009: Rapidly intensifying Hurricane Guillermo (1997). Part I: Low-wavenumber structure and evolution. Mon. Wea. Rev., 137, 603-631, https://doi.org/ 10.1175/2008MWR2487.1.

__ R. Rogers, and S. Lorsolo, 2013: Environmental flow impacts on tropical cyclone structure diagnosed from airborne Doppler radar composites. Mon. Wea. Rev., 141, 2949-2969, https:// doi.org/10.1175/MWR-D-12-00334.1.

Riemer, M., and F. Laliberté, 2015: Secondary circulation of tropical cyclones in vertical wind shear: Lagrangian diagnostic and pathways of environmental interaction. J. Atmos. Sci., 72 , 3517-3536, https://doi.org/10.1175/JAS-D-14-0350.1.

—, M. T. Montgomery, and M. E. Nicholls, 2010: A new paradigm for intensity modification of tropical cyclones: Thermodynamic impact of vertical wind shear on the inflow layer. Atmos. Chem. Phys., 10, 3163-3188, https://doi.org/10.5194/ acp-10-3163-2010.

,-- , and - 2013: Further examination of the thermodynamic modification of the inflow layer of tropical cyclones by vertical wind shear. Atmos. Chem. Phys., 13, 327-346, https:// doi.org/10.5194/acp-13-327-2013.

Rios-Berrios, R., and R. D. Torn, 2017: Climatological analysis of tropical cyclone intensity changes under moderate vertical wind shear. Mon. Wea. Rev., 145, 1717-1738, https://doi.org/ 10.1175/MWR-D-16-0350.1.

Rogers, R., 2010: Convective-scale structure and evolution during a high-resolution simulation of tropical cyclone rapid intensification. J. Atmos. Sci., 67, 44-70, https://doi.org/10.1175/ 2009JAS3122.1.

Rotunno, R., and K. A. Emanuel, 1987: An air-sea interaction theory for tropical cyclones. Part II: Evolutionary study using a nonhydrostatic axisymmetric numerical model. J. Atmos. Sci., 
44, 542-561, https://doi.org/10.1175/1520-0469(1987)044<0542: AAITFT $>2.0 . \mathrm{CO} ; 2$.

Ryglicki, D. R., 2015: An analysis of a barotropically unstable, highRossby number vortex in shear. J. Atmos. Sci., 72, 2152-2177, https://doi.org/10.1175/JAS-D-14-0180.1.

— , and R. E. Hart, 2015: An investigation of center-finding techniques for tropical cyclones in mesoscale models. J. Appl. Meteor. Climatol., 54, 825-846, https://doi.org/10.1175/ JAMC-D-14-0106.1.

— , and D. Hodyss, 2016: A deeper analysis of center-finding techniques for tropical cyclones in mesoscale models. Part I: Low-wavenumber analysis. J. Appl. Meteor. Climatol., 55, 531-559, https://doi.org/10.1175/JAMC-D-15-0125.1.

_ J. H. Cossuth, D. H. Hodyss, and J. D. Doyle, 2018: The unexpected rapid intensification of tropical cyclones in moderate vertical wind shear. Part I: Overview and observations. Mon. Wea. Rev., 146, 3773-3800, https://doi.org/10.1175/MWRD-18-0020.1.

Schecter, D. A., 2015: Response of a simulated hurricane to misalignment forcing compared to the predictions of a simple theory. J. Atmos. Sci., 72, 1235-1260, https://doi.org/10.1175/ JAS-D-14-0149.1.

—-, M. T. Montgomery, and P. D. Reasor, 2002: A theory for the vertical alignment of a quasigeostrophic vortex. J. Atmos. Sci., 59, 150-168, https://doi.org/10.1175/1520-0469(2002) $059<0150$ :ATFTVA $>2.0 . \mathrm{CO} ; 2$.

Shapiro, L. J., and M. T. Montgomery, 1993: A three-dimensional balance theory for rapidly rotating vortices. J. Atmos. Sci., $\mathbf{5 0}$, 3322-3335, https://doi.org/10.1175/1520-0469(1993)050<3322: ATDBTF $>2.0 . \mathrm{CO} ; 2$.

Shuman, F. G., 1957: Numerical methods in weather prediction: II. Smoothing and filtering. Mon. Wea. Rev., 85, 357-361, https:// doi.org/10.1175/1520-0493(1957)085<0357:NMIWPI>2.0.CO;2.

Simpson, R. H., and H. Riehl, 1958: Mid-tropospheric ventilation as a constraint on hurricane development and maintenance. Proc. Tech. Conf. on Hurricanes, Miami, FL, Amer. Meteor. Soc., D4-1-D4-10.

Smith, R. K., and M. T. Montgomery, 2015: Toward clarity on understanding tropical cyclone intensification. J. Atmos. Sci., 72, 3020-3031, https://doi.org/10.1175/JAS-D-15-0017.1.

- W. Ulrich, and G. Sneddon, 2000: On the dynamics of hurricanelike vortices in vertical-shear flows. Quart. J. Roy. Meteor. Soc., 126, 2653-2670, https://doi.org/10.1002/qj.49712656903.

_ J. A. Zhang, and M. T. Montgomery, 2017: The dynamics of intensification in a Hurricane Weather Research and Forecasting simulation of Hurricane Earl (2010). Quart. J. Roy. Meteor. Soc., 143, 293-308, https://doi.org/10.1002/qj.2922.

Stern, D. P., and D. S. Nolan, 2012: On the height of the warm core in tropical cyclones. J. Atmos. Sci., 69, 1657-1680, https:// doi.org/10.1175/JAS-D-11-010.1.

-, and F. Zhang, 2013: How does the eye warm? Part II: Sensitivity to vertical wind shear and a trajectory analysis.
J. Atmos. Sci., 70, 1849-1873, https://doi.org/10.1175/JASD-12-0258.1.

Tang, B., and K. Emanuel, 2010: Midlevel ventilation's constraint on tropical cyclone intensity. J. Atmos. Sci., 67, 1817-1830, https://doi.org/10.1175/2010JAS3318.1.

Tao, D., and F. Zhang, 2015: Effects of vertical wind shear on the predictability of tropical cyclones: Practical versus intrinsic limit. J. Adv. Model. Earth Syst., 7, 1534-1553, https://doi.org/ 10.1002/2015MS000474.

Van Delst, P., 2013: CRTM v2.1.3 user's guide. Joint Center for Satellite Data Assimilation Rep., 232 pp.

Wang, Y., Y. Rao, Z.-M. Tan, and D. Schönemann, 2015: A statistical analysis of the effects of vertical wind shear on tropical cyclone intensity change over the western North Pacific. Mon. Wea. Rev., 143, 3434-3453, https://doi.org/10.1175/ MWR-D-15-0049.1.

Willoughby, H. E., 1990: Temporal changes of the primary circulation in tropical cyclones. J. Atmos. Sci., 47, 242264, https://doi.org/10.1175/1520-0469(1990)047<0242: TCOTPC $>2.0 . \mathrm{CO} ; 2$.

_ F. D. Marks, and R. J. Feinberg, 1984: Stationary and moving convective bands in hurricanes. J. Atmos. Sci., 41, 3189-3211, https://doi.org/10.1175/1520-0469(1984)041<3189: $\mathrm{SAMCBI}>2.0 . \mathrm{CO} ; 2$.

Wirth, V., 2001: Cyclone-anticyclone asymmetry concerning the height of the thermal and the dynamical tropopause. J. Atmos. Sci., 58, 26-37, https://doi.org/10.1175/1520-0469(2001) $058<0026$ :CAACTH $>2.0 . \mathrm{CO} ; 2$.

Xu, K.-M., and D. A. Randall, 2001: Updraft and downdraft statistics of simulated tropical and midlatitude cumulus convection. J. Atmos. Sci., 58, 1630-1649, https://doi.org/10.1175/ 1520-0469(2001)058<1630:UADSOS >2.0.CO;2.

$\mathrm{Xu}, \mathrm{Y}$., and Y. Wang, 2013: On the initial development of asymmetric vertical motion and horizontal relative flow in a mature tropical cyclone embedded in environmental vertical shear. J. Atmos. Sci., 70, 3471-3491, https://doi.org/10.1175/ JAS-D-12-0335.1.

Zawislak, J., H. Jiang, G. R. Alvey III, E. J. Zipser, R. F. Rogers, J. A. Zhang, and S. N. Stevenson, 2016: Observations of the structure and evolution of Hurricane Edouard (2014) during intensity change. Part I: Relationship between the thermodynamic structure and precipitation. Mon. Wea. Rev., 144, 3333-3354, https://doi.org/10.1175/MWR-D-16-0018.1.

Zeng, Z., L. Chen, and Y. Wang, 2008: An observational study of environmental dynamical control of tropical cyclone intensity in the Atlantic. Mon. Wea. Rev., 136, 3307-3322, https://doi.org/ 10.1175/2008MWR2388.1.

Zhang, F., and D. Tao, 2013: Effects of vertical wind shear on the predictability of tropical cyclones. J. Atmos. Sci., 70, 975-983, https://doi.org/10.1175/JAS-D-12-0133.1. 